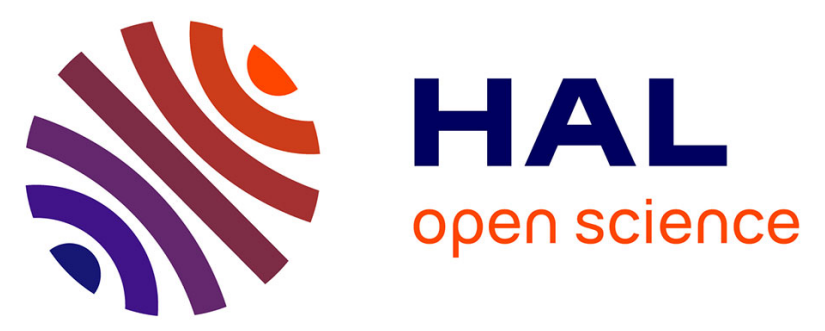

\title{
Répartition des températures dans un tuyau ou un ensemble de tuyaux axisymétriques contenant un fluide en écoulement et soumis à des conditions thermiques dissymétriques
}

A. Cadiou

\section{To cite this version:}

A. Cadiou. Répartition des températures dans un tuyau ou un ensemble de tuyaux axisymétriques contenant un fluide en écoulement et soumis à des conditions thermiques dissymétriques. Revue de Physique Appliquée, 1983, 18 (3), pp.153-168. 10.1051/rphysap:01983001803015300 . jpa-00245080

\author{
HAL Id: jpa-00245080 \\ https://hal.science/jpa-00245080
}

Submitted on 1 Jan 1983

HAL is a multi-disciplinary open access archive for the deposit and dissemination of scientific research documents, whether they are published or not. The documents may come from teaching and research institutions in France or abroad, or from public or private research centers.
L'archive ouverte pluridisciplinaire HAL, est destinée au dépôt et à la diffusion de documents scientifiques de niveau recherche, publiés ou non, émanant des établissements d'enseignement et de recherche français ou étrangers, des laboratoires publics ou privés. 


\title{
Répartition des températures dans un tuyau ou un ensemble de tuyaux axisymétriques contenant un fluide en écoulement et soumis à des conditions thermiques dissymétriques
}

\author{
A. Cadiou (*) \\ Ingénieur de l'Ecole Centrale des Arts et Manufactures, Groupe de Recherches Thermiques du C.N.R.S. (**) \\ Grande Voie des Vignes, 92290 Châtenay-Malabry, France
}

(Reçu le 24 juin 1982, révisé le 19 novembre, accepté le 24 novembre 1982)

\begin{abstract}
Résumé. - Nous présentons ici quelques méthodes pour résoudre le problème thermique suivant. Un tube dans lequel circule un fluide convecteur est soumis à un flux incident extérieur constant selon l'axe du tube mais variable selon la coordonnée angulaire dans une section droite. On calcule les répartitions de température dans le tube et dans le fluide pour deux types de régime d'écoulement : laminaire et turbulent. Dans le premier cas, on étudie l'influence du rayonnement extérieur ainsi que de la convection extérieure au tube sur les températures de paroi et du fluide. L'étude du tube unique isolé est étendue à des tubes adjacents par une de leurs génératrices; elle s'applique aussi dans ce cas à la partie non établie de l'écoulement. Ces calculs ont été appliqués uniquement à titre d'exemple à un sel fondu connu sous le nom d'hitec, constitué de $40 \%$ de $\mathrm{NaNO}_{2}, 53 \%$ de $\mathrm{KNO}_{3}$ et $7 \%$ de $\mathrm{NaNO}_{3}$. Par comparaison, les résultats sont aussi calculés pour le $\mathrm{NaK}$.
\end{abstract}

\begin{abstract}
Different methods to solve the problem of an angle dependent flux on a circular tube where a fluid flows are developped here. We calculate the temperature distribution in the tube wall and in the fluid for two kinds of flow : laminar and turbulent. In the first case, we study the effects of the external radiation and of the convection between the external surface of the tube and the air. The study of an alone tube is extended to several parallel tubes; the non established part of the flow is also studied. The calculations have been applied to a molten salt « hitec " made of $40 \%$ of $\mathrm{NaNO}_{2}, 53 \%$ of $\mathrm{KNO}_{3}$ and $7 \%$ of $\mathrm{NaNO}_{3}$. The results are compared with those calculated for $\mathrm{NaK}$.
\end{abstract}

Nomenclature - Grandeurs géométriques.

$n_{\mathrm{r}}-1$

$\Delta r=\frac{r_{i}}{n_{\mathrm{r}}-1}$

$r_{\mathrm{i}}$

$r_{\mathrm{e}}$

$n_{\mathrm{t}}-1$

$\Delta r_{\mathrm{t}}=\frac{r_{\mathrm{e}}-r_{\mathrm{i}}}{n_{\mathrm{t}}-1}$

$\Delta x$

$\theta$

$n_{\mathrm{u}}-1$

$\Delta \theta=\pi / n_{\mathrm{u}}-1$ pas angulaire

(*) Sur contrat du Ministère de la Recherche et de la Technologie.

$\left({ }^{* *}\right)$ Associé à l'Ecole Centrale des Arts et Manufactures.

pas radial dans le fluide

rayon interne du tube

rayon externe du tube

pas radial dans le tube

pas axial

coordonnée angulaire

nombre de pas angulaires sur $[0, \pi]$

\section{Caractéristiques physiques du fluide}

nombre de pas radiaux dans le fluide pour le calcul numérique

nombre de pas radiaux dans le tube

$$
E
$$

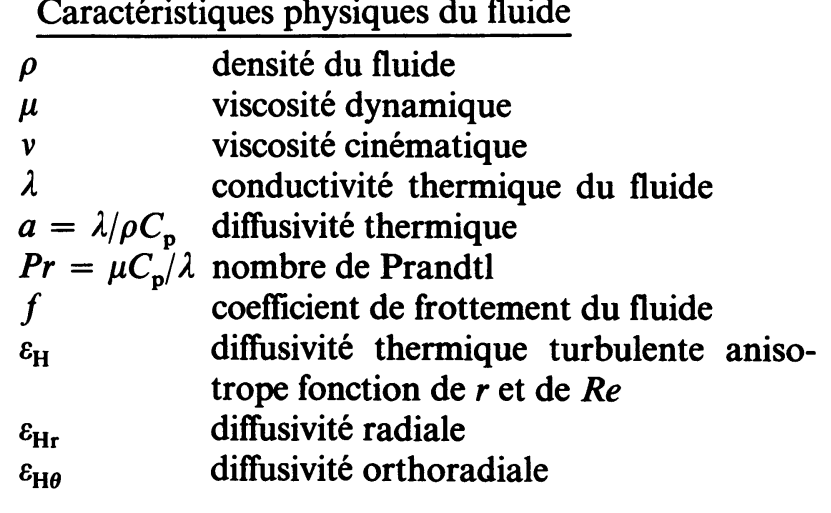

$E=1+\frac{\varepsilon_{\mathrm{H}}}{a} \quad E_{\mathrm{r}}=1+\frac{\varepsilon_{\mathrm{Hr}}}{a} \quad E_{\theta}=1+\frac{\varepsilon_{\mathrm{H} \theta}}{a}$

les $E$ sont sans dimension, fonction de $r$ et de $R e$ seuls, constants en $\theta$

$\varepsilon_{\mathrm{M}} \quad$ diffusivité turbulente de quantité de mouvement. 
Grandeurs ayant une dimension et leurs grandeurs adimensionnées correspondantes

$u$ composante axiale de la vitesse

$v$ composante radiale de la vitesse

$p$ pression du fluide

$F$ température du fluide

$F^{(k)}(i, j)=F(k \Delta x,(i-1) \Delta r,(j-1) \Delta \theta)$

$T$ température du tube

$F^{0}$ grandeur permettant d'adimensionner les températures

$V_{\mathrm{q}}$ vitesse de débit

$x$ coordonnée axiale

$r$ coordonnée radiale

\section{Grandeurs relatives au rayonnement}

$\lambda$ longueur d'onde du rayonnement

$\lambda_{\mathrm{m}}$ longueur d'onde du maximum de luminance énergétique spectrique du corps noir dans le vide

$\varepsilon_{\lambda}$ coefficient d'émission spectral

$L_{\lambda}^{0}$ luminance énergétique spectrique du corps noir dans le vide

$\sigma=5,67 \times 10^{-8}$ U.S.I. constante de Stefan Boltzmann

$Q_{\text {s }}$ flux surfacique du rayonnement direct pris égal à $10^{5} \mathrm{~W} / \mathrm{m}^{2}$ oC pour toutes les applications numériques ici

$\varepsilon$ facteur d'émission du tube dans l'infrarouge

$\alpha$ facteur d'absorption du tube dans le visible ou dans le domaine des gaz de combustion

1. Introduction. - Dans de nombreux cas industriels, des tubes circulaires reçoivent un flux radiatif sur une moitié de leurs génératrices, l'autre moitié étant isolée. On considérera cependant toujours que le plan axial défini par $\theta=0$ constitue un plan de symétrie général (pas de convection naturelle). C'est le cas des capteurs solaires cylindro-paraboliques ou des chaudières à écrans de tubes chauffées au fuel ou encore des centrales solaires à tour. Dans ces deux derniers cas, les tubes ne sont pas uniques mais juxtaposés ou séparés par des ailettes de telle sorte qu'il y a échange radiatif partiel d'un tube à un autre. La symétrie géométrique de révolution se conserve pour l'écoulement mécanique si l'on admet en première approximation que les propriétés physiques du fluide caloporteur interne sont des constantes en fonction de la température. Mais la dissymétrie du flux imposé par l'extérieur implique un champ de température dépendant de la coordonnée angulaire $\theta$ qui définit les différents plans axiaux.

Une première approche consiste à admettre un flux radiatif à rayons parallèles. S'il est plus simple de considérer le cas de la perpendicularité de ces rayons à l'axe, une obliquité connue conduit à des résultats qui se déduisent immédiatement des précédents. Le flux incident affectant le tube peut alors s'écrire : $u^{*}=u / u_{\mathrm{m}}$

$v^{*}=v \operatorname{Re} / 2 u_{\mathrm{m}}$

$p^{*}=2 p / \rho u_{\mathrm{m}}^{2}$

$F^{*}=F / F^{0}$

$T^{*}=T / F^{0}$

$F^{0}=\left(Q_{\mathrm{s}} r_{\mathrm{i}}\right) /(\lambda \pi)$

$\operatorname{Re}=2 r_{\mathrm{i}} V_{\mathrm{q}} / v$

$x^{*}=2 x / \operatorname{Re} r_{\mathrm{i}}$ (adimensionnement classsique dans ces types de problèmes dits de Graetz [12].)

$r^{*}=r / r_{i}$

\section{Indices}

m grandeur moyennée en $r$ et $\theta$

o correspond aux conditions à l'entrée du tube

a signifie atmosphère

$\delta_{\theta}$ vaut 1 pour $0 \leqslant \theta \leqslant \pi / 2$

vaut 0 pour $\pi / 2 \leqslant \theta \leqslant \pi$

$\mathrm{t}$ indique le tube

$\left.{ }^{*}\right)$ grandeur adimensionnée

$h_{\mathrm{e}}$ coefficient de convection entre le tube et le milieu extérieur

\section{Symboles}

$\Delta$ différence de...

$L$ opérateur Laplacien

$Q_{\mathrm{s}} \mathrm{d} s \cos \theta$ pour un élément de surface $\mathrm{d} s$ repéré par l'angle $\theta$,

pour $-\pi / 2 \leqslant \theta \leqslant \pi / 2$,

pour les autres valeurs de $\theta$, le flux est nul.

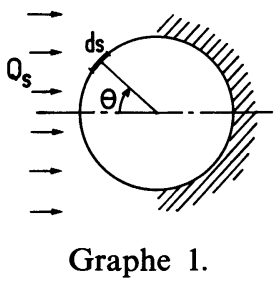

Plusieurs cas peuvent alors se présenter :

- Le fluide intérieur est transparent au rayonnement infrarouge de la paroi interne du tube. Les différences de température entre les génératrices intérieures engendrent des échanges radiatifs qui tendent à uniformiser les températures de cette paroi donc du tube.

- L'enveloppe extérieure est transparente au flux incident et le fluide qui s'écoule à l'intérieur est semitransparent. C'est la technique dite du fluide « noir » utilisée pour certains capteurs solaires.

- Le tube est optiquement opaque et le fluide qui 
s'écoule parallèlement à l'axe du tube, à l'intérieur de celui-ci, l'est également. Le transfert de chaleur dans ce cas, sous l'influence du flux radiatif incident, donne lieu à plusieurs processus élémentaires. Une part du flux radiatif incident est partiellement réfléchie, partiellement transformée en flux radiatif émis, la majeure partie étant transformée par le tube opaque en énergie thermique à la surface, ce qui génère un flux conductif radial et orthloradial à l'intérieur du tube. Le transfert tube-fluide se fait uniquement par convection puisque les fluides considérés sont opaques.

Le premier cas, augmenté du transfert convectif pour le gaz caloporteur, a été traité [7] à Poitiers, le second [8] à Pointe à Pitre.

Le dernier cas fait l'objet du présent travail.

Plusieurs configurations sont envisagées pour le fluide caloporteur : le régime turbulent et l'établissement du régime mécanique et (ou) thermique en laminaire, le régime établi inclus bien entendu.

Dans la première partie, consacrée au régime turbulent, les longueurs d'établissement étant en général très courtes, on a limité l'étude au régime établí. De plus, l'effet conductif de la paroi du tube n'est pas pris en compte. Autrement dit, tout se passe comme si le tube avait une épaisseur nulle. Les résultats obtenus sont donc plus pessimistes que la réalité, le tube n'étant pas là pour homogénéiser les températures par le biais de la composante tangentielle du vecteur flux surfacique.

La deuxième partie a trait au régime laminaire. Il peut sembler paradoxal que, visant des applications où l'on s'efforce de réaliser des écoulements turbulents, on attache une telle importance aux régimes laminaires. L'anomalie n'est qu'apparente. D'abord, parce que de plus en plus on traite thermiquement des fluides extrêmement visqueux ou rhéologiques (plastiques, produits agro-alimentaires à Prandtl très élevé). Mais surtout parce que des régimes laminaires peuvent apparaître occasionnellement lors des mises en route et des arrêts du système. Des difficultés se sont présentées récemment dans nombre d'appareillage, des échangeurs entre autres, lorsque le régime d'écoulement passait par les vitesses caractérisant le régime laminaire. Des avaries allant jusqu'à la destruction par déformation permanente se sont produites du fait de passages trop prolongés par un régime laminaire qui laissait se développer une convection naturelle thermiquement dissymétrisante par rapport aux symétries géométriques. Bien que la situation envisagée ici soit très différente le résultat est le même. Les dissymétries thermiques quelle que soit leur origine introduisent des dilatations différentielles qui en l'absence de toute circulation atteignent leur maximum; elles restent élevées en laminaire et s'atténuent au fur et à mesure que la turbulence tend à homogénéiser le champ de températures.

Dans cette deuxième partie, les cas considérés sont les suivants :

- le flux élémentaire entrant est $Q_{\mathrm{s}} \cos \theta \mathrm{d} S$, l'émit- tance du tube est supposée nulle. Dans ce cas, ont été étudiés l'établissement du régime mécanique et thermique ainsi que le régime établi laminaire;

- l'émission propre du tube est prise en compte. Mais, excepté le cas du capteur solaire cylindroparabolique, le tube est rarement seul dans le système industriel;

- l'étude est généralisée dans une troisième partie au cas d'un tube adjacent par deux génératrices à $180^{\circ}$ à des tubes identiques. L'échange radiatif entre les tubes modifie les résultats précédents;

- la dernière partie tient compte, en outre, des phénomènes de convection entre le milieu extérieur et le tube ou les tubes par le biais du vent extérieur ou d'un écoulement convectif des gaz de combustion d'une chaudière caractérisé par un coefficient convectif $h_{\mathrm{e}}$.

2. Le régime turbulent. - Seule la partie établie du régime est considérée sans qu'il soit tenu compte de l'effet conductif et radiatif du tube. La résolution du problème est essentiellement basée sur le classique bilan énergétique du fluide [1].

Dans cette équation, les diffusivités turbulentes sont remplacées par des valeurs approchées. Ces valeurs sont données par Gärtner [2] et par Stein [5].

2.1 LeS ÉQUATIONS. - Le bilan énergétique conduit à l'équation suivante :

$$
\frac{1}{r} \frac{\partial}{\partial r}\left[r a E_{\mathrm{r}} \frac{\partial F}{\partial r}\right]+\frac{1}{r^{2}} \frac{\partial}{\partial \theta}\left[a E_{\theta} \frac{\partial F}{\partial \theta}\right]=u \frac{\partial F}{\partial x}
$$

avec $E=1+\frac{\varepsilon_{\mathrm{H}}}{a}$ qui est fonction de $r$ et de $\operatorname{Re}\left({ }^{1}\right)$.

Dans cette relation on admet l'hypothèse habituelle suivant laquelle la conduction axiale entraîne des flux négligeables par rapport à ceux de la convection dès que le Reynolds dépasse 100.

Cela est vrai pour les fluides usuels et pour les tubes classiques métalliques à moins que leur épaisseur ne soit très élevée.

Pour des fluides très conducteurs comme les métaux liquides, l'hypothèse n'est toutefois valable que pour des vitesses assez élevées correspondant à des Reynolds supérieurs à 3000 . Or dans ce travail le cas des métaux liquides ne sera abordé qu'en régime turbulent.

La condition à la limite s'écrit :

$$
\lambda\left(\frac{\partial F}{\partial r}\right)_{r=r_{1}}=\delta_{\theta} Q_{s} \cos \theta=q(\theta)
$$

2.2 MéthOde De CALCUI. - 2.2.1 $q(\theta)$ est décomposé en deux termes :

$$
q(\theta)=q_{0}+f(\theta) \text { avec } \int_{0}^{2 \pi} f(\theta) \mathrm{d} \theta=0 .
$$

( ${ }^{1}$ ) Si la diffusivité est un scalaire, les grandeurs équivalentes en régime turbulent $\varepsilon_{\mathrm{Hr}}$ et $\varepsilon_{\mathrm{H} \theta}$ dépendent en toute rigueur de la direction; nous les considèrerons ici comme bidimensionnelles avec deux valeurs caractéristiques suivant la direction radiale ou orthoradiale $\varepsilon_{\mathrm{Hr}}$ et $\varepsilon_{\mathrm{H} \theta}$ respectivement. 
De même pour $F(r, \theta, x)$ :

$$
\begin{aligned}
F(r, \theta, x) & =F_{\mathrm{m}}(x)+\Delta F(r, \theta) \\
& =F_{\mathrm{m}}(x)+\Delta F_{0}(r)+g(r, \theta),
\end{aligned}
$$

où

$$
\Delta F_{0}(r)=\frac{1}{2 \pi} \int_{0}^{2 \pi}\left[F(r, \theta, x)-F_{\mathrm{m}}(x)\right] \mathrm{d} \theta,
$$

et $F_{\mathrm{m}}(x)$ est la température de mélange du fluide à la section $x$.

2.2.2 L'équation (1) devient :

$$
L(\Delta F)=u \frac{\mathrm{d} F_{\mathrm{m}}}{\mathrm{d} x}
$$

et $L(g)=0 ; L$ étant l'opérateur laplacien.

Les conditions aux limites conduisent à :

$$
\begin{aligned}
& q_{0}=\lambda\left(\frac{\mathrm{d} \Delta F_{0}}{\mathrm{~d} r}\right)_{r=r_{\mathrm{i}}} \\
& f(\theta)=\lambda\left(\frac{\partial g}{\partial r}\right)_{r=r_{\mathrm{i}}} .
\end{aligned}
$$

2.2.3 Une série de Fourier représente $f(\theta)$ :

$$
f(\theta)=\sum_{n=1}^{n=\infty}\left(a_{n} \sin n \theta+b_{n} \cos n \theta\right)
$$

d'où :

$$
g(r, \theta)=\frac{r_{\mathrm{i}}}{\lambda} \sum_{n=1}^{\infty} R_{n}\left(\frac{r}{r_{\mathrm{i}}}\right)\left(a_{n} \sin n \theta+b_{n} \cos n \theta\right)
$$

les fonctions $R_{n}$ étant à déterminer.

2.2.4 Les fonctions $R_{n}$ doivent vérifier l'équation :

$$
\frac{\mathrm{d}}{\mathrm{d} r^{*}}\left[E_{\mathrm{r}} r^{*} \frac{\mathrm{d} R_{n}}{\mathrm{~d} r^{*}}\right]-n^{2} \frac{E_{\theta}}{r^{*}} R_{n}=0
$$

avec :

$$
R_{n}^{\prime}(1)=1 \quad \text { et } \quad R_{n}(0)=0
$$

On note : $S_{n}=R_{n}(1)$ pour $n \geqslant 1$ et $S_{0}=2 / N u_{0}$ $\left(N u_{0}\right.$ est la valeur du nombre de Nusselt pour un tube circulaire chauffé de façon uniforme avec la densité de flux $q_{0}: N u_{0}=\left(q_{0} / \Delta F_{0}\left(r_{\mathrm{i}}\right)\right) \times\left(2 r_{\mathrm{i}} / \lambda\right)$. $)$

2.2.5 La température du fluide en $r=r_{\mathrm{i}}$ et en $\theta$ est donnée par:

$$
F\left(r_{\mathrm{i}}, \theta, x\right)=\frac{r_{\mathrm{i}}}{\lambda}\left[S_{0} q_{0}+\sum_{n=1}^{\infty} S_{n}\left(a_{n} \sin n \theta+b_{n} \cos n \theta\right)\right]+F_{\mathrm{m}}(x)
$$

2.2.6 Restent à calculer les $S_{n^{-}}-S_{0}$ est connue à partir de $N u_{0}$.

Pour les autres $S_{n}$, il faut résoudre l'équation différentielle (9).

Le modèle de turbulence utilisé est celui proposé par Gärtner [2] qui tient compte de l'anisotropie en $r$ et $\theta$ de la diffusivité turbulente locale. Ce modèle s'exprime de la manière suivante :

$$
\begin{aligned}
& E_{\mathrm{r}}=1+\frac{\varepsilon_{\mathrm{Mr}}}{v} \times \frac{\varepsilon_{\mathrm{Hr}}}{\varepsilon_{\mathrm{Mr}}} \operatorname{Pr} \\
& \frac{\varepsilon_{\mathrm{Mr}}}{v}=\frac{1}{2}\left\{1+\frac{K^{2}\left(r_{0}^{+}\right)^{2}}{9}\left(1-r^{* 2}\right)^{2}+\left(1+2 r^{* 2}\right)^{2}\left[1-\exp \left(-\frac{1-r^{*}}{A^{+} / r_{0}^{+}}\right)\right]^{2}\right\}^{1 / 2}-\frac{1}{2}
\end{aligned}
$$

avec $A^{+}=26 ; K=0,4 ; r_{0}^{+}=\operatorname{Re} \sqrt{(f / 8)}$ pour $5000 \leqslant R e \leqslant 30000 ; f=0,079 R e^{-0,25}$ $\operatorname{Re} \leqslant 30000 ; f=0,046 \operatorname{Re}^{-0,2}$. $f$ est le coefficient de frottement.

- Le rapport $\frac{\varepsilon_{\mathrm{Hr}}}{\varepsilon_{\mathrm{Mr}}}$ est donné sur la figure 1 .

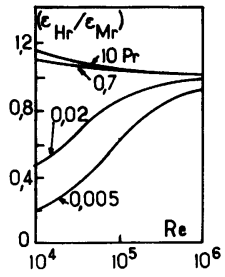

Fig. 1. - Variation de $\varepsilon_{\mathrm{Hr}} / \varepsilon_{\mathrm{Mr}}$ en fonction de $\operatorname{Re}$ pour diffé-

\begin{tabular}{|c|c|c|c|c|c|}
\hline & \multicolumn{5}{|c|}{$\operatorname{Re}$} \\
\hline$P r=10$ & $10^{4}$ & $3 \times 10^{4}$ & $10^{5}$ & $3 \times 10^{5}$ & $10^{6}$ \\
\hline$S_{0}$ & 0,02020 & 0,007778 & 0,002727 & 0,001084 & 0,0003774 \\
\hline$S_{1}$ & 0,02328 & 0,008790 & 0,003048 & 0,001204 & 0,0004154 \\
\hline$S_{2}$ & $0,021 \quad 12$ & 0,008031 & 0,002796 & $0,001 \quad 111$ & 0,0003847 \\
\hline$S_{3}$ & 0,02028 & 0,007747 & 0,002702 & 0,001076 & 0,0003731 \\
\hline$S_{4}$ & 0,01975 & 0,007579 & 0,002646 & 0,001055 & 0,0003663 \\
\hline$S_{5}$ & 0,01935 & 0,007460 & 0,002608 & 0,001041 & 0,0003615 \\
\hline
\end{tabular}
rentes valeurs du nombre de Prandtl utilisé comme paramètre.

[Variation of $\varepsilon_{\mathrm{Hr}} / \varepsilon_{\mathrm{Mr}}$ versus $\dot{R e}$ for different values of the Prandtl number.]
Ces résultats sont aussi donnés sous une autre forme par Stein.

- Le rapport $\frac{\varepsilon_{\mathrm{H} \theta}}{\varepsilon_{\mathrm{Hr}}}$ est donné par : $\frac{\varepsilon_{\mathrm{H} \theta}}{\varepsilon_{\mathrm{Hr}}}=1+\frac{0,2}{1,02-r^{*}}$.

Les fonctions $R_{n}\left(r^{*}\right)$ sont calculées par une méthode numérique.

2.3 LES RÉSUlTATS. - 2.3.1 Les valeurs de $S_{n}$ sont données en fonction de $\operatorname{Re}$ et $\operatorname{Pr}$ pour $n=0 ; 1 ; 2$; $3 ; 4 ; 5$. - Par exemple : 
2.3.2 Si on calcule la série de Fourier de $g(\theta)$ on obtient :

$$
F\left(r_{\mathrm{i}}, \theta, x\right)-F_{\mathrm{m}}(x)=\frac{Q_{\mathrm{s}} r_{\mathrm{i}}}{\lambda}\left[0,318 S_{0}+0,5 S_{1} \cos \theta+0,2126 S_{2} \cos 2 \theta-0,042 S_{4} \cos 4 \theta+\cdots\right] \text {. }
$$

Dans une section du tube, l'écart maximal de température en $r=r_{\mathrm{i}}$ vaut :

$$
\Delta F \triangleq F\left(r_{\mathrm{i}}, 0, x\right)-F\left(r_{\mathrm{i}}, \pi, x\right)=\frac{Q_{\mathrm{s}} r_{\mathrm{i}}}{\lambda} S_{1} .
$$

2.4 Application À L'HiteC eT AU NaK. - Les variations de $\Delta F$ en fonction du nombre de Reynolds sont données sur la figure 2 pour l'hitec et la figure 3 pour le $\mathrm{NaK}$. Le flux solaire concentré environ cent fois est pris égal à $10^{5} \mathrm{~W} / \mathrm{m}^{2}$. Le diamètre du tube est de $15 \mathrm{~mm}$ intérieur.

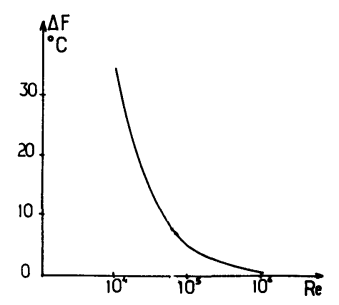

Fig. 2. - Variations de l'écart maximal de température en $r=r_{\mathrm{i}}$ dans une section en fonction du nombre de Reynolds pour de l'hitec dans le cas d'un tube de diamètre $15 \mathrm{~mm}$ et pour un flux incident $Q_{\mathrm{s}}=10^{5} \mathrm{~W} / \mathrm{m}^{2}$.

[Variations of the greatest temperature difference at the tube wall, in a section, versus the Reynolds number for hitec when the tube diameter is $15 \mathrm{~mm}$ and $Q_{s},=10^{5} \mathrm{~W} / \mathrm{m}^{2}$.]

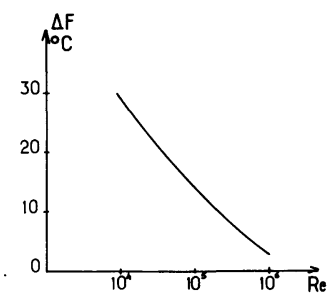

Fig. 3. - Variations de l'écart maximal de température en $r=r_{\mathrm{i}}$ dans une section en fonction du nombre de Reynolds pour du NaK dans le cas d'un tube de diamètre $15 \mathrm{~mm}$ et pour un flux incident $Q_{\mathrm{s}}=10^{5} \mathrm{~W} / \mathrm{m}^{2}$.

[Variations of the greatest temperature difference; at the tube wall, in a section, versus the Reynolds number for $\mathrm{NaK}$ when the tube diameter is $15 \mathrm{~mm}$ and $Q_{\mathrm{s}}=10^{5} \mathrm{~W} / \mathrm{m}^{2}$.]

Le calcul du $\Delta F$ est fait lorsque les deux fluides circulent avec la même vitesse. Les variations de $\Delta F$ avec cette vitesse sont données sur la figure 4 .

Remarque : Ces résultats ont été obtenus en négligeant l'effet du tube. L'écart entre ces résultats théoriques et les faits sera assez faible pour le $\mathrm{NaK}$ dont la conductivité est supérieure à celle de l'acier. Avec l'hitec, cette discordance sera plus élevée mais le

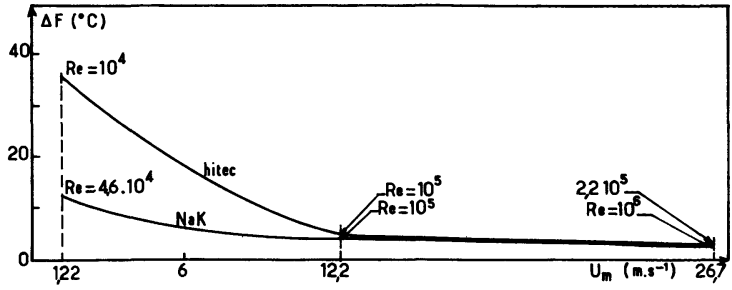

Fig. 4. - Valeurs de $\Delta F$ en fonction de la vitesse du fluide pour de l'hitec et du $\mathrm{NaK}$ (propriétés physiques du fluide prises à $300^{\circ} \mathrm{C}$ ) pour un flux incident de $10^{5} \mathrm{~W} / \mathrm{m}^{2}$ et un tube de diamètre $15 \mathrm{~mm}$.

[Values of $\Delta F$ versus the fluid velocity for hitec and $\mathrm{NaK}$ (the physical properties of the fluid are given at $300^{\circ} \mathrm{C}$ ) for an incident flux $Q_{\mathrm{s}}=10^{5} \mathrm{~W} / \mathrm{m}^{2}$ and for $r_{\mathrm{i}}=7.5 \mathrm{~mm}$.]

calcul conduit à une restructuration des écarts de température.

3. Le régime laminaire stationnaire. $-3.1 \mathrm{CON}$ VECTION FLUIDE-TUBE ET CONDUCTION DANS LE TUBE. La première partie est consacrée à l'établissement du régime qui se produit sur une distance non négligeable et la seconde au régime mécaniquement et thermiquement établi.

3.1.1 Etude de la région d'établissement. - Les développements du régime mécanique et du régime thermique sont supposés commencer dès l'entrée.

3.1.1.1 Les équations et les conditions aux limites mécaniques. - Le profil des vitesses obéit à :

- l'équation de Navier-Stokes :

$$
u \frac{\partial u}{\partial x}+v \frac{\partial u}{\partial r}=-\frac{1}{\rho} \frac{\mathrm{d} p}{\mathrm{~d} x}+\frac{v}{r} \frac{\partial}{\partial r}\left(r \frac{\partial u}{\partial r}\right)
$$

- l'équation de continuité :

$$
\frac{\partial u}{\partial x}+\frac{1}{r} \frac{\partial}{\partial r}(r v)=0 .
$$

Les hypothèses habituelles sont admises. tantes.

- Les propriétés physiques du fluide sont cons-

- La pression est uniforme dans une section droite du tube.

- La vitesse axiale est indépendante de $r$ dans la section d'entrée du tube.

Les conditions aux limites s'écrivent :

- $u=v=0$ pour $r=r_{\mathrm{i}}$,

- $\left(\frac{\partial u}{\partial r}\right)_{r=0}=0$,

- pour $x=0, u=u_{\mathrm{m}}, v=0, p=p_{0}$. 
Cet ensemble d'équations implique que la symétrie de révolution est conservée du point de vue de la mécanique des fluides. Entre autres les vitesses créées par la convection naturelle n'interviennent pas. Les équations mécaniques sont découplées du champ thermique.

3.1.1.2 Les équations et les conditions aux limites thermiques. - Pour le profil de températures dans le tube et dans le fluide, compte tenu des transferts de chaleur envisagés et des conditions aux limites dissymétriques on a :

- l'équation de la chaleur dans le fluide

$$
u \frac{\partial F}{\partial x}+v \frac{\partial F}{\partial r}=a\left(\frac{\partial^{2} F}{\partial r^{2}}+\frac{1}{r} \frac{\partial F}{\partial r}+\frac{1}{r^{2}} \frac{\partial^{2} F}{\partial \theta^{2}}\right)
$$

- dans le tube

$$
\frac{\partial^{2} T}{\partial r^{2}}+\frac{1}{r} \frac{\partial T}{\partial r}+\frac{1}{r^{2}} \frac{\partial^{2} T}{\partial \theta^{2}}=0 .
$$

Pour des raisons déjà exposées les conductions axiales sont négligées [11].

Les conditions aux limites associées sont :

- sur la surface externe du tube

$$
\lambda_{\mathrm{t}}\left(\frac{\partial T}{\partial r}\right)_{r=r_{\mathrm{e}}}=\delta_{\theta} Q_{\mathrm{s}} \cos \theta
$$

- à l'interface fluide-paroi :

en

$$
\begin{aligned}
r & =r_{\mathrm{i}} \\
F & =T
\end{aligned}
$$

et

$$
\lambda\left(\frac{\partial F}{\partial r}\right)_{r=r_{i}}=\lambda_{t}\left(\frac{\partial T}{\partial r}\right)_{r=r_{i}}
$$

- à l'entrée du tube, en $x=0$

$$
F=F_{0} \text {. }
$$

Dans ces équations, on remplace les grandeurs utilisées par leurs grandeurs adimensionnées équivalentes (voir nomenclature) afin de faire apparaître les paramètres du système et pour simplifier l'écriture les "*» ont été supprimées par la suite.

3.1.1.3 Traitement des équations. - La méthode utilisée pour résoudre le profil des vitesses est la méthode donnée par Manohar [3]. Quant au profil de températures, il est résolu par une méthode aux différences finies : pour les dérivées axiales, on a utilisé des différences finies aval et pour les dérivées radiales des différences centrées sauf pour le terme $v \frac{\partial F}{\partial r}$.

Pour celui-ci :

si $v(r)<0$

$$
\frac{\partial F}{\partial r}=\frac{F(r+\Delta r)-F(r)}{\Delta r}
$$

si $v(r)>0$

$$
\frac{\partial F}{\partial r}=\frac{F(r)-F(r-\Delta r)}{\Delta r}
$$

Les équations deviennent alors:

- pour le fluide

$$
F^{(k+1)}(i, j)=F A \cdot F^{(k)}(i, j)+F B \cdot F^{(k)}(i-1, j)+F C \cdot F^{(k)}(i+1, j)+F D \cdot\left[F^{(k)}(i, j+1)+F^{(k)}(i, j-1)\right]
$$

avec (pour $v<0)$ :

$$
\begin{aligned}
& F A=1+\frac{v(r)}{u(r)} \frac{\Delta x}{\Delta r}-\frac{2 \Delta x}{u(r) \operatorname{Pr}}\left(\frac{1}{\Delta r^{2}}+\frac{1}{r^{2} \Delta \theta}\right) \\
& F B=\frac{\Delta x}{\Delta r} \frac{1}{\operatorname{Pr} u(r)}\left(\frac{1}{\Delta r}-\frac{1}{2 r}\right) \\
& F C=\frac{\Delta x}{u(r) \Delta r}\left(-v(r)+\frac{1}{\operatorname{Pr}}\left(\frac{1}{\Delta r}+\frac{1}{2 r}\right)\right) \\
& F D=\frac{\Delta x}{u(r)} \frac{1}{\operatorname{Pr}} \frac{1}{r^{2}(\Delta \theta)^{2}} .
\end{aligned}
$$

Un bilan d'énergie au centre du fluide conduit à :

$$
F^{(k+1)}(1, j)=2 Z_{u}\left[F^{(k)}(2,1)+2 \sum_{i_{u}=2}^{n_{u}-1} F^{k}\left(2, i_{u}\right)+F^{k}\left(2, n_{u}\right)\right]+\left(1-4 Z_{u}\left(n_{u}-1\right)\right) F^{k}(1, j)
$$

avec $Z_{u}=\frac{\Delta x}{\Delta r} \frac{\Delta \theta}{\operatorname{Pr}} \frac{1}{\pi u(1)}$ 
- pour le tube

avec

$$
-2 T A \cdot T(i, j)+T B \cdot T(i+1, j)+T C \cdot T(i-1, j)+T D \cdot[T(i, j+1)+T(i, j-1)]=0
$$

$$
\begin{aligned}
T A=\frac{1}{\Delta r_{\mathrm{t}}^{2}}+\frac{1}{r^{2} \Delta \theta^{2}} & T B=\frac{1}{\Delta r_{\mathrm{t}}^{2}}+\frac{1}{2 r \Delta r_{\mathrm{t}}} \\
T C=\frac{1}{\Delta r_{\mathrm{t}}^{2}}-\frac{1}{2 r \Delta r_{\mathrm{t}}} & T D=\frac{1}{r^{2} \Delta \theta^{2}} .
\end{aligned}
$$

Pour $i=n_{\mathrm{t}}$

$$
-2 T A \cdot T\left(n_{\mathrm{t}}, j\right)+\frac{2}{\Delta r_{\mathrm{t}}^{2}} \cdot T\left(n_{\mathrm{t}}-1, j\right)+T D \cdot\left[T\left(n_{\mathrm{t}}, j+1\right)+T\left(n_{\mathrm{t}}, j-1\right)\right]=-2 \Delta r_{\mathrm{t}} \frac{\lambda}{\lambda_{\mathrm{t}}} \pi \delta_{\theta} \cos \theta \times T B .
$$

Pour $i=1$ :

$$
T(1, j)=F\left(n_{r}, j\right)=\frac{\left(\lambda_{t} / \Delta r_{t}\right) T(2, j)+(\lambda / \Delta r) F\left(n_{r}-1, j\right)}{\left(\lambda_{t} / \Delta r_{t}\right)+(\lambda / \Delta r)}
$$

Ces équations pour le tube peuvent s'écrire sous forme matricielle : $X T . T=X C$ où $T(i, j)=T(i+$ $\left.(j-1) n_{\mathbf{t}}\right)$.

3.1.1.4 Méthode de calcul. - On suppose le profil de températures connu en $x=k . \Delta x$.

- On calcule $u(i)$ et $v(i)$ pour $1 \leqslant i \leqslant n_{r}$.

- (19) donne $F^{k+1}(i, j)$ pour $2 \leqslant i \leqslant n_{r-1}$,

$$
1 \leqslant j \leqslant n_{u} .
$$

- Connaissant tous les $F^{k+1}\left(n_{r-1}, j\right)$ on a le vecteur $X C$ et on peut donc résoudre le système $X T . T=$ $X C$ et donc calculer les températures dans le tube.

- (23) nous donne $F^{k+1}\left(n_{r}, j\right)$.

- Et enfin (20) donne $F^{k+1}(1, j)$.

Le profil de température étant connu en $x=0$, cette méthode permet de calculer toutes les températures voulues.

3.1.1.5 Les résultats. - Le but essentiel est de déterminer les écarts maximaux de température qui peuvent apparaître dans le tube.

Néanmoins, on donne ici d'autres résultats qui ont leur importance.

\section{Pour les vitesses :}

La vitesse axiale adimensionnée $u^{*}$ ne dépend que de $x^{*}$ et de $r^{*}$. Elle est indépendante des propriétés du fluide.

Cette courbe nous donne la longueur d'établissement du régime mécanique : $x^{*}=0,2$, c'est-à-dire qu'on retrouve bien :

$$
L_{\mathrm{M}}=0,05 \operatorname{Red}_{i}
$$

en grandeur réelle.

\section{Pour les températures:}

- Un des résultats les plus importants est que la grandeur adimensionnée $F^{*}\left(x^{*}, r^{*}, \theta\right)-F^{*}\left(0, r^{*}, \theta\right)$ à une abscisse donnée dépend du nombre de Prandtl du fluide, de sa conductivité ainsi que de celle de la paroi.

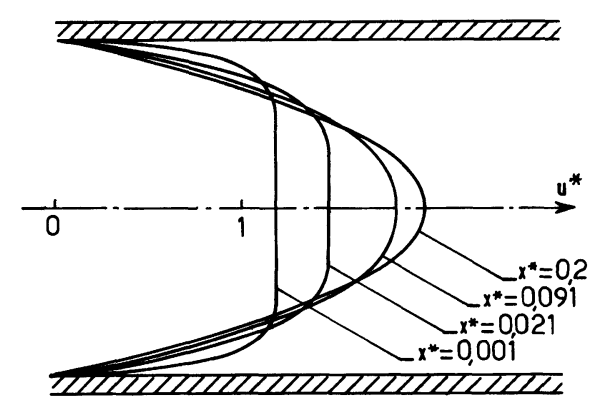

Fig. 5. - Variations de la vitesse axiale adimensionnée $u^{*}$ en fonction de $r^{*}$ pour différentes sections : $x^{*}=0,001$; $x^{*}=0,021 ; x^{*}=0,091 ; x^{*}=0,2$.

[Variations of the non dimensional axial velocity $u^{*}$ versus $r^{*}$ for different sections : $x^{*}=0.001 ; x^{*}=0.021 ; x^{*}=0.091$; $x^{*}=0.2$.]

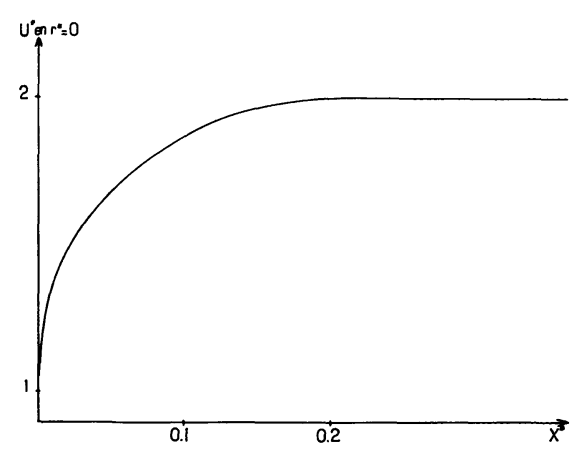

Fig. 6. - Evolution de la vitesse axiale adimensionnée au centre du fluide avec la coordonnée axiale $x^{*}$.

[Variation of the non dimensional axial velocity $u^{*}$ at the center of the fluid, with the axial coordonate $x^{*}$.] 


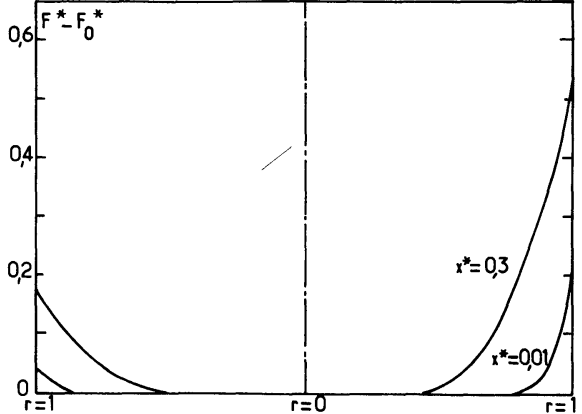

Fig. 7. - Graphe de la température du fluide le long du diamètre repéré par $\theta=0$ et $\theta=\pi$ pour différentes valeurs de $x^{*}$ et pour de l'hitec. $\operatorname{Pr}=10 ; \lambda=0,5 \mathrm{~W} / \mathrm{m}{ }^{\circ} \mathrm{C} ; \lambda_{\mathrm{t}}=$ $25 \mathrm{~W} / \mathrm{m}{ }^{\circ} \mathrm{C}$.

[Values of the hitec temperature along the diameter of a section $\theta=0$, and $\theta=\pi$ for different values of $x^{*}: \operatorname{Pr}=10$ $\lambda=0.5 \mathrm{~W} / \mathrm{m}^{\circ} \mathrm{C} ; \lambda_{\mathrm{t}}=25 \mathrm{~W} / \mathrm{m}^{\circ} \mathrm{C}$.]

- L'écart de température entre le point le plus chaud et le plus froid de la surface externe du tube dans une section augmente avec la .coordonnée axiale $x^{*}$ jusqu'à atteindre une valeur constante correspondant au régime établi (Fig. 8).

Pour l'hitec avec un tube 15-18 en acier inoxydable, on obtient une longueur d'établissement du régime égale à :

$$
L_{\mathrm{T}}=0,225 \operatorname{Red}_{i} .
$$

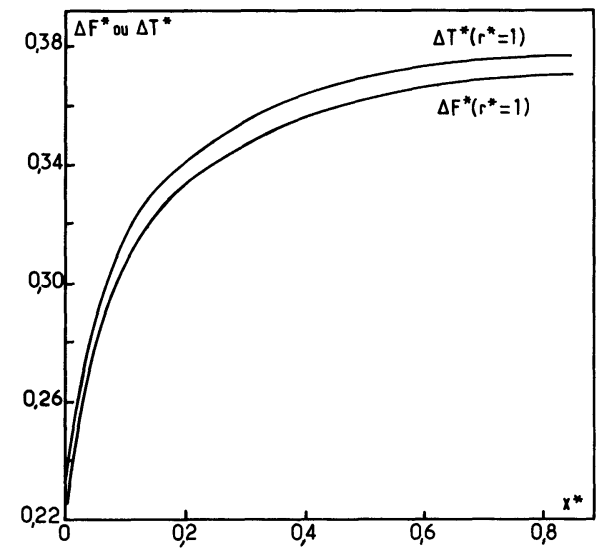

Fig. 8. - Variations de $\Delta F^{*}=F^{*}(\theta=0)-F^{*}(\theta=\pi)$ pour $r^{*}=1$ et de $\Delta T^{*}=T^{*}(\theta=0)-T^{*}(\theta=\pi)$ pour $r^{*}=r_{\mathrm{e}} / r_{\mathrm{i}}$ en fonction de $x^{*}$.

[Variations de $\Delta F^{*}=F^{*}(\theta=0)-F^{*}(\theta=\pi)$ for $r^{*}=1$ and of $\Delta T^{*}=T^{*}(\theta=0)-T^{*}(\theta=\pi)$ for $r^{*}=r_{\mathrm{e}} / r_{\mathrm{i}}$ versus $x^{*}$.]

Naturellement des extensions peuvent être aisément effectuées pour différentes valeurs des paramètres $\operatorname{Pr}, \lambda$ et $\lambda_{\text {r }}$.

On donne à titre d'exemple la variation des écarts $\operatorname{maximaux} \Delta T^{*}=\Delta T \times\left(\lambda \pi / Q_{\mathrm{s}} r_{\mathrm{i}}\right)$ à la surface externe du tube en fonction de $x^{*}=2 x / R e . r_{\mathrm{i}}$, paramétrée avec le nombre de Prandtl (Fig. 9); on remarquera néanmoins que les conductivités du fluide et du tube

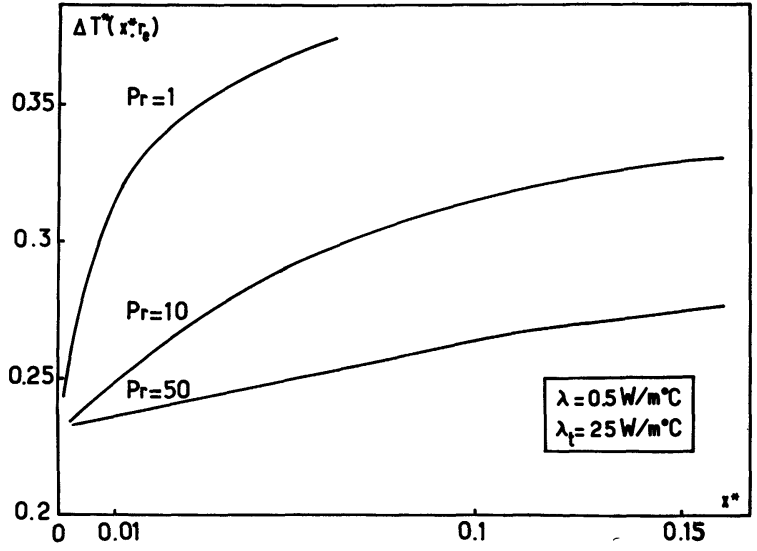

Fig. 9. - Variations de $\Delta T^{*}=T^{*}\left(\theta=0, r^{*}=r_{\mathrm{e}} / r_{\mathrm{i}}\right)$ $T^{*}\left(\theta=\pi, r_{\mathrm{e}} / r_{\mathrm{i}}\right)$ en fonction de la distance axiale adimensionnée $x^{*}$ pour $\lambda=0,5 \mathrm{~W} / \mathrm{m}^{\circ} \mathrm{C}$ et $\lambda_{\mathrm{t}}=25 \mathrm{~W} / \mathrm{m}{ }^{\circ} \mathrm{C}$ et pour différentes valeurs du nombre de Prandtl :

$$
\begin{aligned}
& -\operatorname{Pr}=1, \quad \text { c'est-à-dire } \mu C_{\mathrm{p}}=0,5 \text { U.S.I. } \\
& \text { - } P r=10 \text {, c'est-à-dire } \mu C_{\mathrm{p}}=5 \quad \text { U.S.I. } \\
& \text { - } P r=50 \text {, c'est-à-dire } \mu C_{\mathrm{p}}=25 \text { U.S.I. }
\end{aligned}
$$

[Variations of $\Delta T^{*}=T^{*}\left(\theta=0, r^{*}=r_{\mathrm{e}} / r_{\mathrm{i}}\right)-T^{*}(\theta=$ $\left.\pi, r^{*}=r_{\mathrm{e}} / r_{\mathrm{i}}\right)$ versus $x^{*}$ for different values of $\operatorname{Pr}$ and for $\lambda=0.5 \mathrm{~W} / \mathrm{m}^{\circ} \mathrm{C}$ and $\lambda_{\mathrm{t}}=25 \mathrm{~W} / \mathrm{m}^{\circ} \mathrm{C}$.]

sont constantes, de telle sorte qu'il faut comprendre $\operatorname{Pr}$ comme variant uniquement avec $\mu . C_{\mathrm{p}}$.

Sur la figure 10 c'est le nombre de Prandtl qui est considéré comme une constante, le tube a toujours

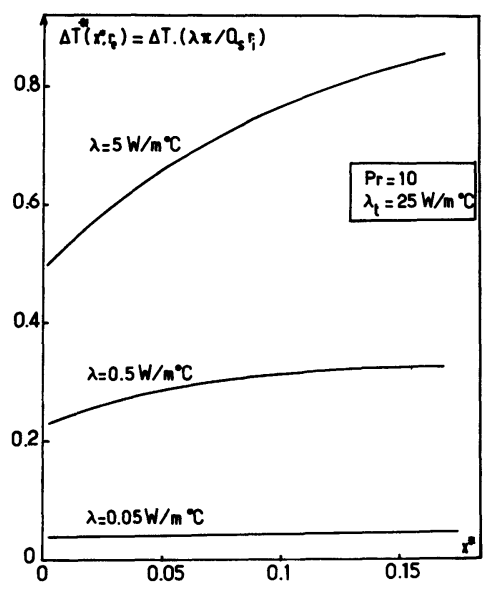

Fig. 10. - Variations de $\Delta T^{*}=T^{*}\left(\theta=0, r^{*}=r_{\mathrm{e}} / r_{\mathrm{i}}\right)$ $T^{*}\left(\theta=\pi, r^{*}=r_{\mathrm{e}} / r_{\mathrm{i}}\right)$ pour plusieurs valeurs de la conductivité du fluide $\lambda$ avec $P r=10$ et $\lambda_{\mathrm{t}}=25 \mathrm{~W} / \mathrm{m}^{\circ} \mathrm{C}$.

[Variations of $\Delta T^{*}=T^{*}\left(\theta=0, r_{\mathrm{e}} / r_{\mathrm{i}}\right)-T^{*}\left(\theta=\pi, r_{\mathrm{e}} / r_{\mathrm{i}}\right)$ for different values of the fluid conductivity with $P r=10$ and $\lambda_{\mathrm{t}}=25 \mathrm{~W} / \mathrm{m}^{\circ} \mathrm{C}$.]

une conductivité de $25 \mathrm{~W} / \mathrm{m}^{\circ} \mathrm{C}$, mais cette fois le paramètre est la conductivité du fluide $\lambda$.

La figure 11 quant à elle montre pour un fluide donné et à caractéristiques géométriques identiques l'influence de la conductivité du tube $\lambda_{\text {t }}$.

Enfin la dernière figure (Fig. 12), donne des résultats plus immédiatement interprétables car elle fournit à un facteur multiplicatif près l'écart maximal de températures réel, le paramètre étant cette fois la nature du fluide. 


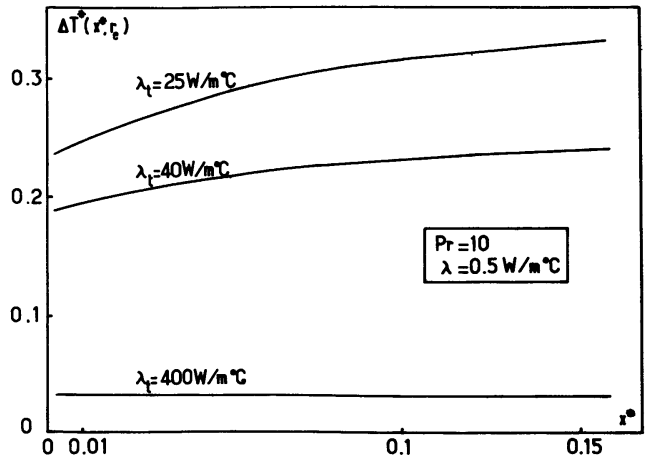

Fig. 11. - Variations de $\Delta T^{*}=T^{*}\left(\theta=0, r_{\mathrm{e}} / r_{\mathrm{i}}\right)-T^{*}(\theta=$ $\left.\pi, r_{\mathrm{e}} / r_{\mathrm{i}}\right)$ pour plusieurs valeurs de la conductivité du tube $\lambda_{\mathrm{t}}$ avec $\operatorname{Pr}=10$ et $\lambda=0,5 \mathrm{~W} / \mathrm{m}{ }^{\circ} \mathrm{C}$.

[Variations of $\Delta T^{*}=T^{*}\left(\theta=0, r_{\mathrm{e}} / r_{\mathrm{i}}\right)-T^{*}\left(\theta=\pi, r_{\mathrm{e}} / r_{\mathrm{i}}\right)$ for different values of the tube conductivity $\lambda_{\mathrm{t}}$ with $P r=10$ and $\lambda=0.5 \mathrm{~W} / \mathrm{m}^{\circ} \mathrm{C}$.]

3.1.2 Etude du régime établi. - Le traiter en tant que limite du problème précédent nécessite un temps de calcul excessif aussi est-il préférable d'employer une autre méthode. Dans le bilan d'énergie du fluide, on introduit $v=0$ et $u(r)=2 u_{\mathrm{m}}\left(1-\left(r / r_{\mathrm{i}}\right)^{2}\right)$ et on

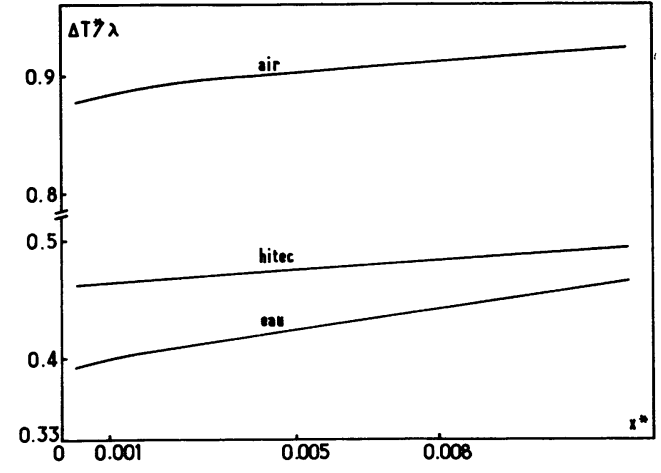

Fig. 12. - Variation de $\frac{\Delta T^{*}}{\lambda}=\Delta T \cdot \frac{\pi}{Q_{\mathrm{s}} r_{\mathrm{i}}}$ pour plusieurs fluides :

— air à $150^{\circ} \mathrm{C} \quad \operatorname{Pr}=0,686 \lambda=0,035 \mathrm{~W} / \mathrm{m}^{\circ} \mathrm{C}$

— hitec à $300^{\circ} \mathrm{C} \quad \operatorname{Pr}=10 \quad \lambda=0,5 \mathrm{~W} / \mathrm{m}^{\circ} \mathrm{C}$

— eau à $60^{\circ} \mathrm{C} \quad \operatorname{Pr}=3 \quad \lambda=0,651 \mathrm{~W} / \mathrm{m}^{\circ} \mathrm{C}$ $\lambda_{\mathrm{t}}=25 \mathrm{~W} / \mathrm{m}^{\circ} \mathrm{C}$.

[Variations of $\frac{\Delta T^{*}}{\lambda}=\Delta T \cdot \frac{\pi}{Q_{\mathrm{s}} r_{\mathrm{i}}}$ for a few fluids :

- air at $150^{\circ} \mathrm{C} ;$ - hitec at $300^{\circ} \mathrm{C}$; - water at $60^{\circ} \mathrm{C}$.]

résoud par une méthode de séparation des variables. Ceci conduit au résultat suivant :

$$
F^{*}\left(x, r^{*}, \theta\right)=F_{\mathrm{m}}^{*}(0)+4 \frac{r_{\mathrm{e}}}{r_{\mathrm{i}}^{2}} \frac{1}{\operatorname{Pr} \operatorname{Re}} x+2 \frac{r_{\mathrm{e}}}{r_{\mathrm{i}}}\left[\frac{1}{2} r^{* 2}\left(1-\frac{r^{* 2}}{4}\right)-\frac{7}{48}\right]+\sum_{n \geqslant 1} A_{n} \cos n \theta
$$

avec :

$$
A_{n}=-2\left(\frac{r_{\mathrm{i}}}{r_{\mathrm{e}}}\right)^{n-1} \frac{\cos \frac{n \pi}{2}}{1-a_{\mathrm{p}}\left(\frac{r_{\mathrm{i}}}{r_{\mathrm{e}}}\right)^{2 n}} \frac{\lambda}{\lambda_{\mathrm{t}}} \frac{1-a_{\mathrm{p}}}{n\left(n^{2}-1\right)}
$$

avec $a_{\mathrm{p}}=\frac{\left(\lambda_{\mathrm{t}} / \lambda\right)-1}{\left(\lambda_{\mathrm{t}} / \lambda\right)+1}$

Pour ces valeurs de $r_{\mathrm{i}}, r_{\mathrm{e}}, \lambda$ et $\lambda_{\mathrm{t}}$ (valeurs utilisées pour la Fig. 13), l'écart maximum de température pour $r^{*}=1$, adimensionné est de 0,371 . Pour de l'hitec et avec un flux $Q_{\mathrm{s}}$ de $10^{5} \mathrm{~W} / \mathrm{m}^{2}$ (ce qui correspond à une concentration de $100 \mathrm{du}$ rayonnement solaire), l'écart réel de température est de $170^{\circ} \mathrm{C}$, évidemment incompatible avec la tenue du tube.

Fig. 13. - Courbes d'isovaleur de $F^{*}(x)-F_{m}^{*}(x)$ dans une section pour un tube $\varnothing 15 / 18 \lambda=0,5 \mathrm{~W} / \mathrm{m}^{\circ} \mathrm{C}$; $\lambda_{\mathrm{t}}=25 \mathrm{~W} / \mathrm{m}^{\circ} \mathrm{C}$.

[Curve of constant $F^{*}(x)-F_{m}^{*}(x)$ in a section for : $r_{\mathrm{i}}=7.5 \mathrm{~mm} ; r_{\mathrm{e}}=9 \mathrm{~mm} ; \lambda_{\mathrm{t}}=25 \mathrm{~W} / \mathrm{m}{ }^{\circ} \mathrm{C} ; \lambda=0.5 \mathrm{~W} / \mathrm{m}^{\circ} \mathrm{C}$. $]$

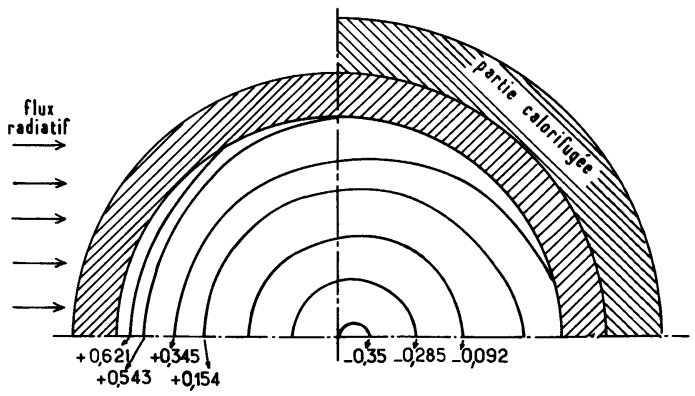

3.2 EMISSION EXTERNE DU TUBE. - Les phénomènes radiatifs à la surface externe du tube sont pris en compte. Toujours pour diminuer les temps de calcul, on suppose le régime des vitesses établi lorsque le tube est chauffé. La paroi externe du tube est supposée à émission et à absorption isotropes. Deux types de tube sont étudiés.

Dans un premier cas, le tube en inox est recouvert d'une peinture (pyromark 2500) qui permet d'assimiler la surface externe du tube à un corps noir dans les longueurs d'onde considérées (le visible et l'infrarouge). Dans 
un deuxième cas, on utilise un tube en acier oxydé qui émet dans l'infrarouge avec $\varepsilon_{\mathrm{IR}}=0,25$ et qui absorbe dans le visible avec $\alpha_{\text {vis. }}=0,6$.

Ces résultats proviennent d'études effectuées au G.R. 14 du C.N.R.S. associé à l'E.C.P. par P. Stekelorom et A. Soufiani [9].

D'autre part nous tenons compte de l'effet de l'atmosphère.

Le coefficient d'émission monochromatique de l'atmosphère peut en première approximation s'exprimer par :

$$
\begin{aligned}
& \left.\begin{array}{rl}
\text { pour } & 0 \leqslant \lambda \leqslant 8 \mu \mathrm{m} \\
\text { et } & \lambda \geqslant 12 \mu \mathrm{m}
\end{array}\right\} \varepsilon_{\lambda}=1, \\
& \text { pour } 8 \mu \mathrm{m} \leqslant \lambda \leqslant 12 \mu \mathrm{m} \quad \varepsilon_{\lambda}=0 \text {. }
\end{aligned}
$$

Le flux émis par l'atmosphère à la température $T_{\mathrm{a}}$ est donc égal à :

$$
\Phi_{\text {atm. }}=\pi \int_{\lambda=0}^{\lambda=\infty} \varepsilon_{\lambda} L_{\lambda}^{0}\left(T_{\mathrm{a}}\right) \mathrm{d} \lambda .
$$

Le calcul de ce terme utilise les fonctions

qui sont tabulées [13].

$$
z\left(\lambda / \lambda_{\mathrm{m}}\right)=\left[\int_{0}^{\lambda} \pi \varepsilon_{\lambda^{\prime}} L_{\lambda^{\prime}}^{0}\left(T_{\mathrm{a}}\right) \mathrm{d} \lambda^{\prime}\right] / \sigma T_{\mathrm{a}}^{4}
$$

D'où :

$$
\Phi_{\text {atm. }}=\sigma T_{\mathrm{a}}^{4}\left[z\left(8 / \lambda_{\mathrm{m}}\right)+\left(1-z\left(12 / \lambda_{\mathrm{m}}\right)\right)\right]
$$

avec $\lambda_{\mathrm{m}} T_{\mathrm{a}} \simeq 2898 \mu \mathrm{mK}$.

Des modèles plus précis existent pour $\varepsilon_{\lambda}$, mais étant donné la petite part qu'a le flux atmosphérique dans le flux incident, l'utilisation d'un modèle approché est sufisante. établi :

3.2.1 Les équations du système. - Le profil des vitesses est connu puisqu'on suppose le régime mécanique

$$
\begin{aligned}
& u(r)=2 u_{\mathrm{m}}\left(1-\left(\frac{r}{r_{\mathrm{i}}}\right)^{2}\right), \\
& v(r)=0 .
\end{aligned}
$$

En ce qui concerne les températures, toutes les équations du paragraphe 1.1.1 restent valables sauf l'équation à la limite en $r=r_{\mathrm{e}}$ qui doit être remplacée par :

$$
-\lambda_{\mathrm{t}}\left(\frac{\partial T}{\partial r}\right)_{r=r_{e}}=\delta_{\theta}\left[\varepsilon \sigma T^{4}-\alpha\left(0,75 \sigma T_{\mathrm{a}}^{4}+Q_{\mathrm{s}} \cos \theta\right)\right] .
$$

3.2.2 Le traitement des équations. - On utilise les grandeurs adimensionnées citées dans la nomenclature. En vue de linéariser les équations, nous avons utilisé une méthode préconisée par Ness [6]. Elle consiste à remplacer $T^{* 4}$ par :

$$
T^{* 4}\left(r_{\mathrm{e}}^{*}, \theta\right)=T_{0}^{* 3}\left(r_{\mathrm{e}}^{*}, \theta\right)\left[4 T^{*}\left(r_{\mathrm{e}}^{*}, \theta\right)-3 T_{0}^{*}\left(r_{\mathrm{e}}^{*}, \theta\right)\right],
$$

où $T_{0}^{*}\left(r_{\mathrm{e}}^{*}, \theta\right)$ est une valeur approximative de $T^{*}\left(r_{\mathrm{e}}^{*}, \theta\right)$.

Aussi, en appliquant les différences finies et en utilisant les mêmes notations qu'au paragraphe 1.1.2, l'équation pour $i=n_{\mathrm{t}}$ s'écrit :

$$
\{-2 T A-\beta T B\} T\left(n_{t}, j\right)+\frac{2}{\Delta r_{t}^{2}} T\left(n_{t}-1, j\right)+T D\left\{T\left(n_{t}, j+1\right)+T\left(n_{t}, j-1\right)\right\}=-\gamma T A
$$

avec :

$$
\begin{aligned}
& \beta=8 \delta_{\theta} \varepsilon \Delta r_{\mathrm{t}} \alpha T_{0}^{3} / \lambda_{\mathrm{t}} \\
& \gamma=2 \delta_{\theta} \Delta r_{\mathrm{t}}\left[l\left(3 \varepsilon T_{0}^{4}+0,75 \alpha T_{\mathrm{a}}^{4}\right)+\alpha \lambda \pi \cos \theta\right] / \lambda_{\mathrm{t}} \\
& l=\left(\frac{Q_{\mathrm{s}} r_{\mathrm{d}}}{\lambda \pi}\right)^{3} \sigma r_{\mathrm{i}} .
\end{aligned}
$$

Les autres équations sont identiques.

La méthode numérique utilisée pour résoudre le problème groupe une méthode de différences finies et une méthode itérative. 
L'organigramme du calcul est le suivant :

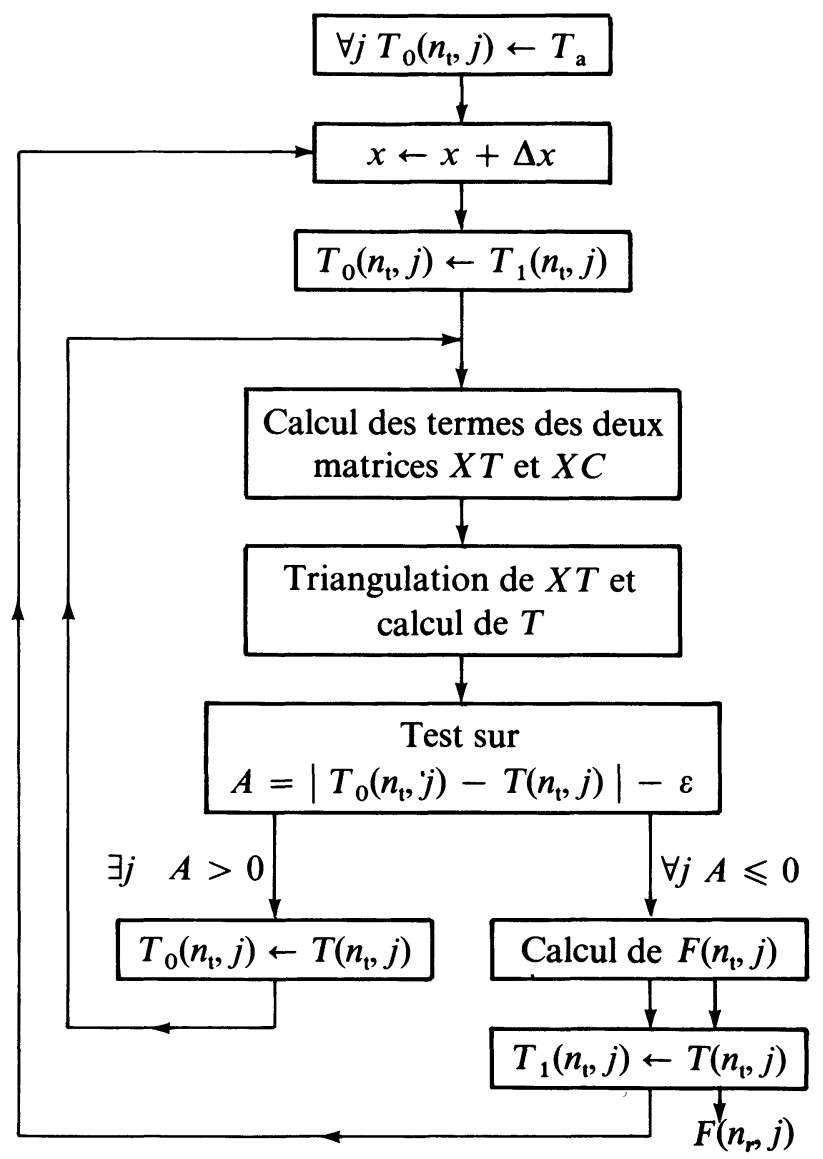

3.2.3 Les résultats pour un tube. - Les paramètres du système sont plus nombreux que dans le cas précédent.

Nous avons :

- $\lambda, P_{\mathrm{r}}, \lambda_{\mathrm{t}}, \Delta r_{\mathrm{t}}$

- $r_{\mathrm{i}}, F_{0}$ (la température d'entrée du fluide), $Q_{\mathrm{s}}, T_{\mathrm{a}}$ (la température ambiante) $\varepsilon$ et $\alpha$.

L'application du programme de calcul à l'hitec conduit aux résultats suivants :

- Les écarts maximaux de température à la surface externe du tube dans une section sont beaucoup plus faibles que dans le cas précédent (voir Fig. 14) car les effets de l'émission propre du tube sont loin d'être négligeables.

Les courbes correspondent à trois cas particuliers :

a) ne tient pas compte de l'émission du tube, le flux solaire incident est de $10^{5} \mathrm{~W} / \mathrm{m}^{2}$;

b) le tube émet comme un corps noir, le flux incident est également $10^{5} \mathrm{~W} / \mathrm{m}^{2}$;

c) le tube est en acier oxydé mais, pour comparer des conditions comparables, le flux incident est tel que le flux absorbé en surface par le tube soit le même que dans le cas précédent, ce qui conduit à

$$
Q_{\mathrm{s}}=\frac{1}{0,6} \times 10^{5} \mathrm{~W} / \mathrm{m}^{2} \text {. }
$$

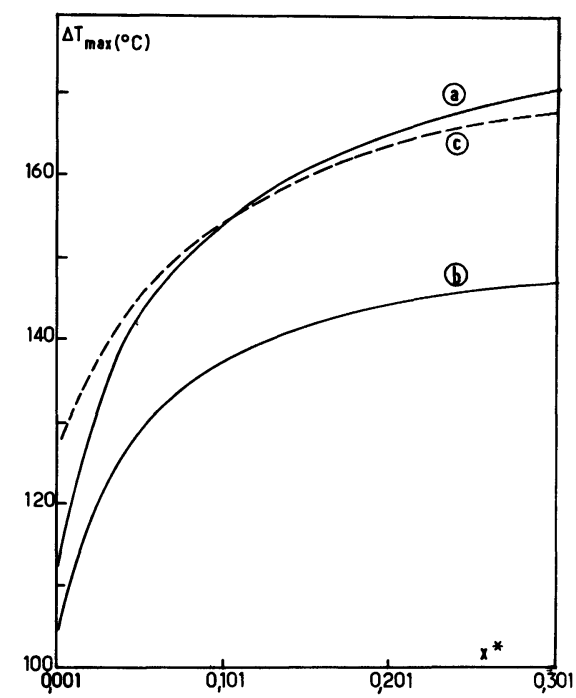

Fig. 14. - Valeurs de l'écart maximal de températures dans une section en $r=r_{\mathrm{e}}$ en fonction de $x^{*}$ pour de l'hitec et pour: $F_{0}=200^{\circ} \mathrm{C} ; T_{\mathrm{a}}=20^{\circ} \mathrm{C}$; tube $15-18 ; \lambda_{\mathrm{t}}=25 \mathrm{~W} / \mathrm{m}^{\circ} \mathrm{C}$ dans les trois cas $\mathrm{a}, \mathrm{b}$, et $\mathrm{c}$ cités précédemment.

[Values of the greatest difference of temperatures in a section for $r=r_{\mathrm{e}}$ versus $x^{*}$ for hitec and for: $F_{0}=200^{\circ} \mathrm{C} ; T_{\mathrm{a}}=20^{\circ} \mathrm{C}$; tube $\varnothing 15 / 18 ; \lambda_{\mathrm{t}}=25 \mathrm{~W} / \mathrm{m}{ }^{\circ} \mathrm{C}$ in the three cases $\mathrm{a}, \mathrm{b}$, and $\mathrm{c}$ given above.]

- Les isothermes dans le fluide tendent vers l'axisymétrie (voir Fig. 15, Fig. 16).

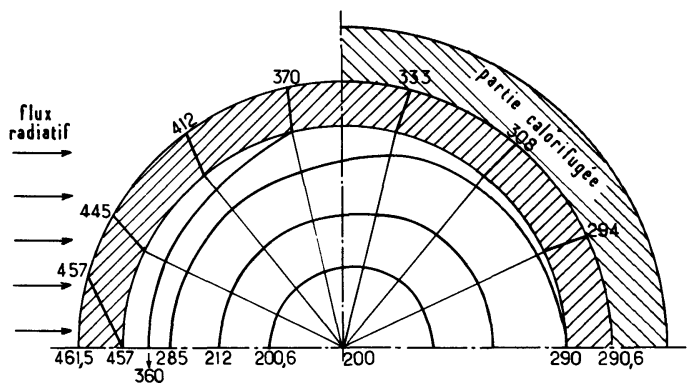

Fig. 15. - Isothermes dans le fluide et dans la paroi du tube dans la section $x^{*}=0,311$ (le régime peut être considéré comme établi) lorsque l'émission du tube n'est pas prise en compte. Le fluide est l'hitec : $R e=2000$; $F_{0}=200^{\circ} \mathrm{C} ; Q_{\mathrm{s}}=10^{5} \mathrm{~W} / \mathrm{m}^{2} ;$ tube $\varnothing 15-18$ en acier inoxydable.

[Isotherms in the fluid and in the tube wall in the section $x^{*}=0.311$ (the flow is established) when the tube emission is not considered. The fluid used is hitec : $R e=2000$; $F_{0}=200{ }^{\circ} \mathrm{C} ; Q_{\mathrm{s}}=10^{5} \mathrm{~W} / \mathrm{m}^{2}$; tube $\varnothing 15-18$ made of stainless iron.] 


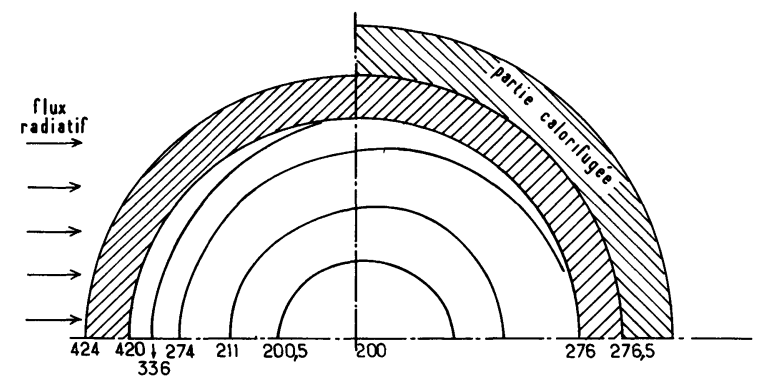

Fig. 16. - Isothermes dans le fluide dans la section $x^{*}=0,311$ lorsque la surface externe du tube émet avec un facteur d'émission $\varepsilon=1$. Le fluide est l'hitec dans les mêmes conditions que pour la figure précédente.

[Isotherms in the fluid in the section $x^{*}=0.311$ when the emission factor of the external part of the tube $\varepsilon$ is equal to 1 . The fluid used is hitec under the same conditions as in figure 10.]

Pour donner un caractère plus général à ces résultats, nous avons également étudié le cas de tubes placés en parallèle et juxtaposés. La surface externe des tubes est ici assimilée à un corps noir.
3.3 Tubes ADJACENTS. - Le schéma du système est le suivant :

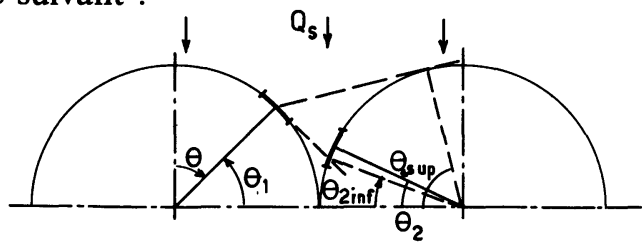

Graphe 2.

La surface élémentaire $\mathrm{d} s_{1}$ (bande de longueur unité de largeur $r_{\mathrm{e}} \mathrm{d} \theta_{1}$ ) émet mais elle reçoit également une partie du rayonnement émis par $\mathrm{d} s_{2}$ lorsque $\theta_{2}$ caractérisant la position de $\mathrm{d} s_{2}$ est compris entre deux angles limites.

Aussi, dans le calcul, intervient ce qu'on appelle un facteur de forme entre ces deux types de surface.

Sa valeur est la suivante [10] :

$$
\mathrm{d} f_{\mathrm{d} s_{1} \mathrm{~d} s_{2}}=\frac{1}{2} \frac{|r \mathrm{~d} a-a \mathrm{~d} r|}{r^{2}}
$$

avec

$$
\begin{aligned}
a & =r_{\mathrm{e}}\left(2 \sin \theta_{1}-\sin \left(\theta_{1}+\theta_{2}\right)\right) \\
\mathrm{d} a & =-r_{\mathrm{e}} \cos \left(\theta_{1}+\theta_{2}\right) \mathrm{d} \theta_{2} \\
r & =2 r_{\mathrm{e}}\left(\frac{1}{2}\left(1+\cos \left(\theta_{1}+\theta_{2}\right)\right)+1-\cos \theta_{1}-\cos \theta_{2}\right) \\
\mathrm{d} r & =\frac{r_{\mathrm{e}}^{2}}{r}\left(2 \sin \theta_{2}-\sin \left(\theta_{1}+\theta_{2}\right)\right) \mathrm{d} \theta_{2} .
\end{aligned}
$$

Les valeurs limites de $\theta_{2}$ pour $\theta_{1}$ fixé sont :

$$
\begin{aligned}
& \theta_{2 \text { inf. }}=\frac{\pi}{2}-\theta_{1}-\operatorname{Arcsin}\left(2 \cos \theta_{1}-1\right) \\
& \theta_{2 \text { sup. }}=\operatorname{Arc} \sin \left(\frac{\sin \theta_{1}+2\left(2-\cos \theta_{1}\right) \sqrt{1-\cos \theta_{1}}}{5-4 \cos \theta_{1}}\right) .
\end{aligned}
$$

3.3.1 Les équations et leur traitement. - La condition à la limite en $r=r_{\mathrm{e}}$ devient :

$$
-\lambda_{\mathrm{t}}\left(\frac{\partial T}{\partial r}\right)_{r=r_{\mathrm{e}}}=\delta_{\theta}(-E+J)
$$

avec : la radiosité $J=\sigma T^{4}(\theta)$, l'éclairement

$$
E=0,75 \sigma T_{\mathrm{a}}^{4}+Q_{\mathrm{s}} \cos \theta+\int_{\theta_{2} \text { inf. }}^{\theta_{2} \text { sup. }} \sigma T^{4}\left(\theta_{2}\right) \mathrm{d} f_{\mathrm{ds}_{1} \mathrm{~d} s_{2}} .
$$

On a supposé, en écrivant cela, que toute une bande d $s_{2}$ est à la même température $T\left(\theta_{2}\right)$. On adimensionne cette équation.

$T^{* 4}(\theta)$ est linéarisé comme au paragraphe précédent.

Le terme $T^{* 4}\left(\theta_{2}\right)$ dans l'intégrale sera remplacé par son terme approché.

Aussi, pour $i=n_{\mathrm{t}}$ on obtient une équation ayant la même forme que celle obtenue en 2.1 .2 avec $\alpha=\varepsilon=1$. La seule différence est l'expression de $\gamma$ qui devient ici :

avec :

$$
\gamma=2 \delta_{\theta} \frac{\Delta r_{\mathrm{t}}}{\lambda_{\mathrm{t}}}\left[l\left(3 T_{0}^{4}+0,75 T_{\mathrm{a}}^{4}+\sum_{j=j_{\text {inf }}}^{j=j_{\text {sup }}-1} \frac{F(j)+F(j+1)}{2} \Delta \theta\right)+\lambda \pi \cos \theta\right]
$$

$$
F(j)=\sigma T^{4}\left(\theta_{2}\right) \frac{|r \mathrm{~d} a-a \mathrm{~d} r|}{r^{2} \mathrm{~d} \theta}
$$




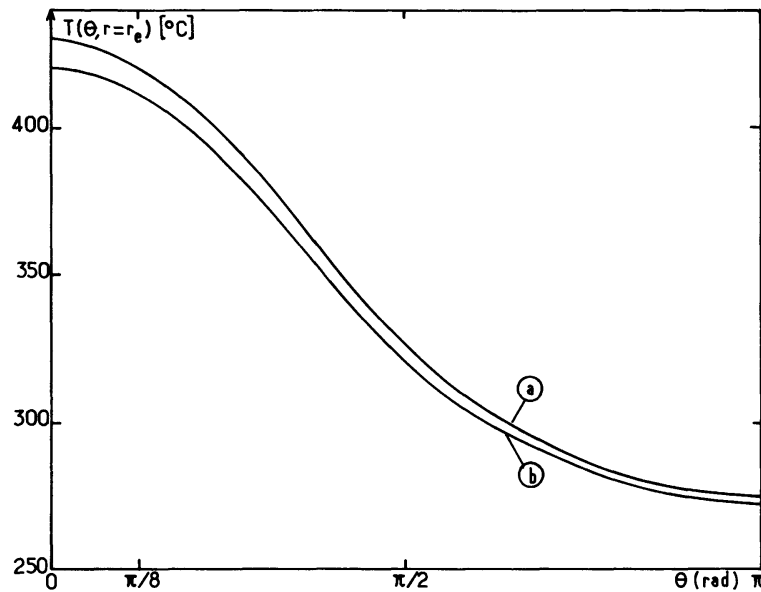

Fig. 17. - Courbes donnant la variation de la température externe du tube avec la coordonnée angulaire $\theta$ dans la section $x^{*}=0.3$ dans les deux cas suivants : (a) plusieurs tubes en parallèle, (b) un tube unique, pour de l'hitec dans les conditions d'utilisation usuelles.

[Variation of the external temperature of the tube with $\theta$ in the section $x^{*}=0,3$ in the two following cases : (a) several parallel tubes, (b) a single tube, for hitec in the usual use conditions.]

Ceci constitue la seule transformation à effectuer pour résoudre le problème.

L'organigramme du calcul est le même qu'en 3.2.2.

3.3.2 Les résultats. - Les tubes adjacents vont avoir tendance à chauffer le tube considéré. On constate une élévation du niveau thermique du tube, cette élévation étant plus sensible dans la partie chauffée que dans la partie isolée. Pour l'hitec, dans les conditions prises généralement pour le calcul

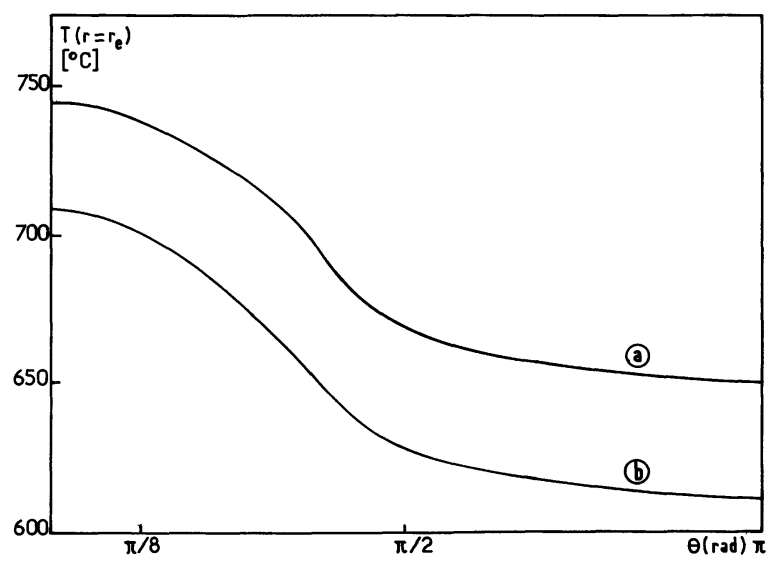

Fig. 18. - Courbes donnant la variation de la température externe du tube avec la coordonnée angulaire $\theta$ dans la section $x^{*}=0.3$ dans les deux cas suivants : (a) plusieurs tubes en parallèle, (b) un tube unique, pour de l'air avec $F_{0}=400 \mathrm{~K} ; T_{\mathrm{a}}=20^{\circ} \mathrm{C} ; Q_{\mathrm{s}}=10^{5} \mathrm{~W} / \mathrm{m}^{2} ;$ tube $\varnothing 15 / 18$ en inox; $R e=2000$.

[Variation of the external temperature of the tube with $\theta$ in the section $x^{*}=0.3$ in the two following cases : (a) several parallel tubes, (b) an alone tube, for air with : $F_{0}=$ $400 \mathrm{~K} ; T_{\mathrm{a}}=20^{\circ} \mathrm{C} ; Q_{\mathrm{s}}=10^{5} \mathrm{~W} / \mathrm{m}^{2}$; tube $\varnothing 15 / 18$ made of stainless iron; $R e=2000$.]

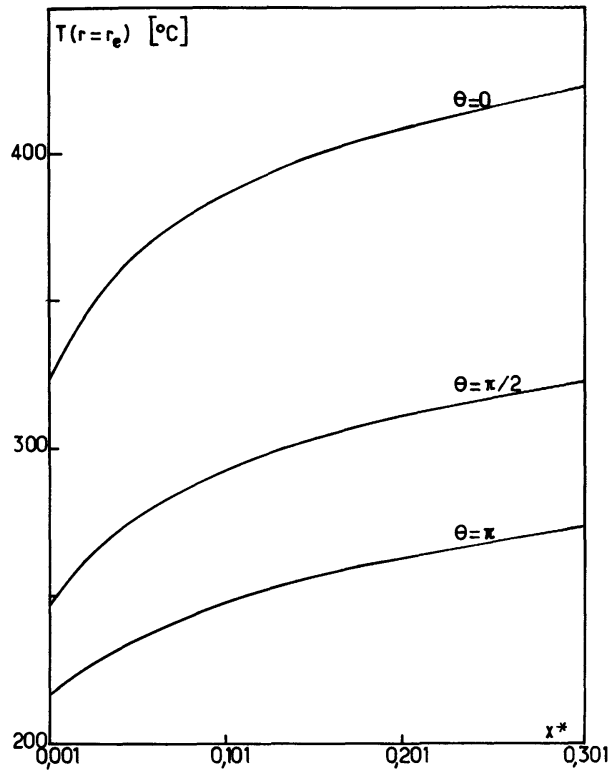

Fig. 19. - Evolution de la température extérieure du tube avec $x^{*}$ sur trois génératrices correspondant à $\theta=0$, $\theta=\pi / 2$ et $\theta=\pi$ dans le cas de plusieurs tubes en parallèle avec de l'hitec dans les conditions usuelles.

[Variations of the external temperature of the tube with $x^{*}$ for 3 generating lines corresponding to $\theta=0, \theta=\pi / 2$ and $\theta=\pi$ in the case of several parallel tubes, with hitec, in the usual conditions.]

$\left(F_{0}=200^{\circ} \mathrm{C}, T_{\mathrm{a}}=20^{\circ} \mathrm{C}, R e=2000, Q_{\mathrm{s}}=10^{5} \mathrm{~W} / \mathrm{m}^{2}\right.$, tube en acier inox de diamètre 15-18, de conductivité $25 \mathrm{~W} / \mathrm{m}^{\circ} \mathrm{C}$ ), l'écart de température entre le point le plus chaud et le plus froid de la surface externe du tube dans une section est à peu près le même pour un tube seul et pour plusieurs tubes. Par contre, les températures sont différentes.

Les résultats sont présentés sous forme de courbes :

- La figure 17 montre dans la section $x^{*}=0,3$ la variation des températures à la surface du tube dans le cas d'un tube et dans celui de plusieurs tubes pour de l'hitec avec les valeurs usuelles des paramètres nécessaires au calcul.

- La figure 18 donne les mêmes courbes pour l'air avec $F_{0}=400 \mathrm{~K}, T_{\mathrm{a}}=20^{\circ} \mathrm{C}, Q_{\mathrm{s}}=10^{5} \mathrm{~W} / \mathrm{m}^{2}$ et le même tube que pour l'hitec.

- La figure 19 donne pour l'hitec l'évolution de $T\left(r_{\mathrm{e}}, \theta\right)$ avec $x$ pour trois valeurs de $\theta: 0, \frac{\pi}{2}, \pi$.

- La figure 20 donne $T\left(r_{\mathrm{e}}, x^{*}\right)$, en fonction de l'angle $\theta$ pour plusieurs valeurs de $x^{*}$ dans le cas de l'hitec.

3.4 EFFet DE LA CONVECTION AVEC Le MilieU EXTÉRIEUR. - Des mouvements de convection (vent, gaz de combustion) peuvent avoir beaucoup d'influence sur les répartitions de température dans le tube.

Le modèle convectif utilisé ici admet un coefficient de convection $h_{\mathrm{e}}$ constant sur la partie non isolée.

Du point de vue résolution du problème, il suffit, 


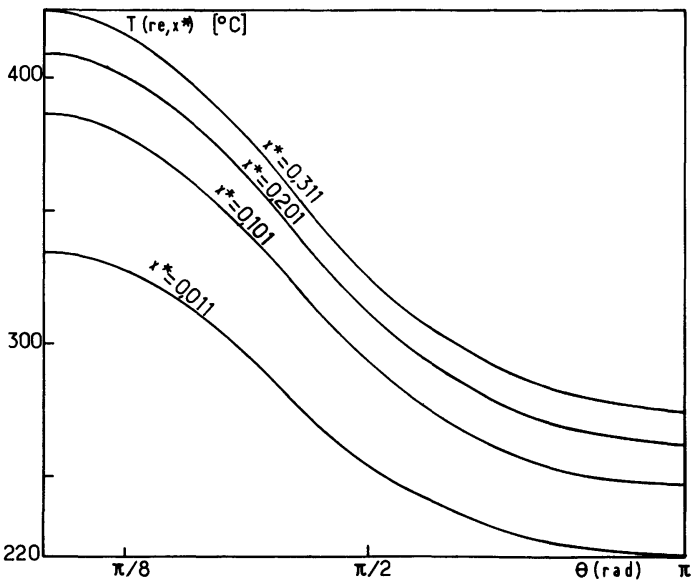

Fig. 20. - Valeurs de la température de la surface externe du tube en fonction de l'angle dans une section pour différentes sections dans le cas de plusieurs tubes en parallèle, pour de l'hitec.

[Values of the temperature of the external surface of the tube versus $\theta$ in a section, for a few sections, in the case of several tubes for hitec.]

dans l'équation à la limite externe, de faire intervenir le flux convectif.

Dans le cas d'un tube seul par exemple, elle devient :

$$
\begin{aligned}
& -\lambda_{\mathrm{t}}\left(\frac{\partial T}{\partial r}\right)_{r=r_{\mathrm{e}}}= \\
= & \delta_{\theta}\left[\sigma T^{4}-Q_{\mathrm{s}} \cos \theta-0,75 \sigma T_{\mathrm{a}}^{4}+h_{\mathrm{e}}\left(T-T_{\mathrm{a}}\right)\right] .
\end{aligned}
$$

Lors de la discrétisation du problème, les équations conservent la même forme. La seule différence étant

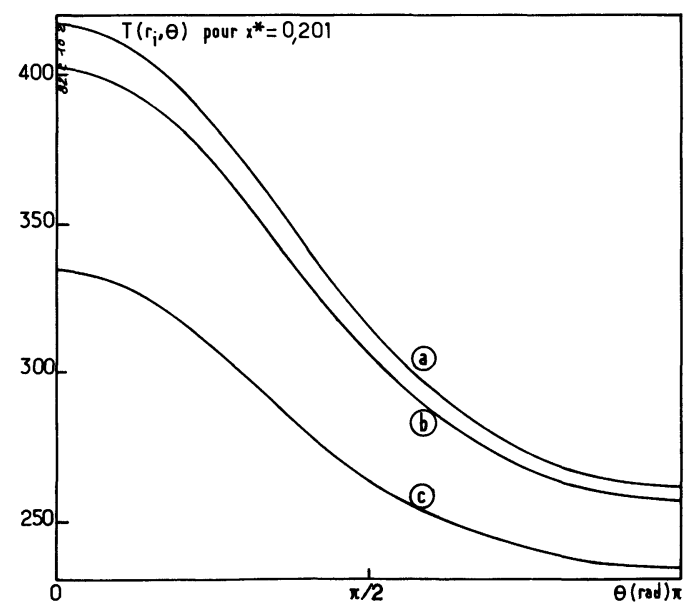

Fig. 21. - Variations de la température de la surface interne du tube avec $\theta$ dans la section $x^{*}=0.201$ pour différentes valeurs du coefficient de convection externe $h_{\mathrm{e}}$ : (a) $h_{\mathrm{e}}=0 \mathrm{~W} / \mathrm{m}^{2}{ }^{\circ} \mathrm{C}$, (b) $h_{\mathrm{e}}=15 \mathrm{~W} / \mathrm{m}^{2}{ }^{\circ} \mathrm{C}$, (c) $h_{\mathrm{e}}=$ $100 \mathrm{~W} / \mathrm{m}^{2}{ }^{\circ} \mathrm{C}$, pour de l'hitec.

[Variations of the temperature of the internal surface of the tube with $\theta$ in the section $x^{*}=0,201$ for different values of the external heat transfer coefficient $h_{\mathrm{e}}$ : (a) $h_{\mathrm{e}}=0 \mathrm{~W} / \mathrm{m}^{2}{ }^{\circ} \mathrm{C}$, (b) $h_{\mathrm{e}}=15 \mathrm{~W} / \mathrm{m}^{2}{ }^{\circ} \mathrm{C}$, (C) $h_{\mathrm{e}}=100 \mathrm{~W} / \mathrm{m}^{2}{ }^{\circ} \mathrm{C}$, for hitec.] qu'au terme noté $\gamma$, il faudra ajouter $2 \delta_{\theta} \frac{\Delta r_{\mathrm{t}}}{\lambda_{\mathrm{t}}} r_{\mathrm{i}} h_{\mathrm{e}} T_{\mathrm{a}}^{*}$ et au terme noté $\beta, 2 \delta_{\theta} \frac{\Delta r_{\mathrm{t}}}{\lambda_{\mathrm{t}}} r_{\mathrm{i}} h_{\mathrm{e}}$.

Quant aux résultats, la convection avec un milieu extérieur plus froid a évidemment tendance à atténuer l'élévation de température de la région la plus chaude. Appliqué à de l'hitec dans les conditions habituelles $\left(F_{0}=200^{\circ} \mathrm{C}, T_{\mathrm{a}}=20^{\circ} \mathrm{C}, Q_{\mathrm{s}}=10^{5} \mathrm{~W} / \mathrm{m}^{2}\right.$, tube $15-$ 18 en inox), on obtient les profils de température représentés sur la figure 21 où le paramètre est la valeur de $h_{\mathrm{e}}$ depuis 0 jusqu'à 100 en passant par 15 .

Le traitement convectif est donné ici dans un cas simple et classique : le refroidissement sur une moitié du tube par un coefficient convectif $h_{\mathrm{e}}$ constant.

Rien n'empêche d'utiliser le même programme pour des $h_{\mathrm{e}}$ variables en $\theta$ à condition de connaître la fonction $h_{\mathrm{e}}(\theta)$.

Le ralentissement que provoquent les parois dans les intervalles droits entre deux tubes consécutifs peut donc être pris en compte. Rien n'oblige non plus à limiter à un refroidissement l'action de la convection extérieure qui peut, au contraire, ajouter un flux convectif à un flux radiatif.

4. Extensions possibles et conclusion - Les résultats de cette étude montrent l'importance qu'il faut accorder aux dissymétries de flux qui peuvent provoquer des écarts très grands de température dans une même section du tube.

Un exemple numérique mieux que n'importe quelle conclusion générale illustre l'importance des processus retenus :

$-Q_{\mathrm{s}}=10^{5} \mathrm{~W} / \mathrm{m}^{2}$,

- tube $15-18 \mathrm{~mm}$ en acier inoxydable recouvert de peinture noire pour le flux incident,

- fluide : hitec,

- température d'entrée du fluide : $200^{\circ} \mathrm{C}$,

$-R e=2000$.

Les écarts de température entre deux génératrices peuvent atteindre $150^{\circ} \mathrm{C}$.

Il faudra donc veiller, lors de l'exploitation du système, à ce que de tels régimes ne puissent exister nulle part dans le réseau de tubes car ils pourraient déformer définitivement l'ensemble. Une étude plus complète devrait tenir compte du temps. Si le passage par des régimes laminaires dans des mises en route et arrêt se fait en un temps faible devant les temps caractéristiques de conduction-convection, les écarts seront réduits.

Quoi qu'il en soit du caractère limite des résultats cités, on a établi un code de calcul qui permet de calculer les températures du fluide et surtout de la paroi du tube pour un flux incident radiatif à rayons parallèles tombant perpendiculairement sur un ou plusieurs tubes à axes parallèles et adjacents les uns aux autres. En régime stationnaire, le flux absorbé est emporté dans la majeure partie par l'écoulement (établi ou non) laminaire ou turbulent d'un fluide 
opaque. Le fluide extérieur peut à volonté provoquer une perte par convection s'il est plus froid que le tube ou un apport qui s'ajoute à $Q_{\mathrm{s}}$ s'il est plus chaud.

Le code s'adapte à toutes les épaisseurs et rayons de tube, à tous les fluides opaques et à toutes les distributions de flux pourvu qu'elles soient constantes en $x$.

Néanmoins, lors de l'utilisation de ce code, certaines transformations doivent être faites si on change de fluide, de tube ou de conditions aux limites. Dans les deux premiers cas de transformations, il faut vérifier que le pas axial $\Delta x$ est suffisamment petit pour obtenir des résultats significatifs. La condition essentielle à vérifier est que :

$$
F A=1-2 \frac{\Delta x}{u(r) \operatorname{Pr}}\left(\frac{1}{\Delta r^{2}}+\frac{1}{r^{2} \Delta \theta}\right)>0 .
$$

Dans les trois cas de transformation, il faut s'assurer, comme dans toutes les utilisations des différences finies, que les pas $\Delta r$ et $\Delta \theta$ sont corrects.

La méthode la plus efficace pour s'assurer du bienfondé du choix des pas est de diviser par deux les pas choisis et de comparer les résultats dont l'identité valide les valeurs choisies.

Physiquement l'attaque dissymétrique d'un tube de révolution par le biais des conditions aux limites aboutit à des résultats identiques aux dissymétries créées par la convection naturelle bien que par un processus complètement différent. Dans cette dernière la symétrie géométrique et aux limites peut être rompue par des composantes radiales de vitesses issues des différences de densités elles-mêmes générées par des flux même symétriques aux parois. En convection naturelle les dilatations différentielles entre génératrices ont abouti à des dilatations permanentes d'échangeurs donc à leur destruction. Les dissymétries de flux aux parois conduisent à des résultats identiques.

Autre point commun à signaler, l'influence néfaste des dissymétries des conditions aux limites ou de la convection naturelle est effacée par une convection forcée virulente où des fluctuations de vitesse tendent à homogénéiser le mélange et à atténuer les dissymétries thermiques. Dans les deux cas les régimes turbulents « de croisière » pour lesquels sont calculés les appareils ne sont donc que rarement dangereux. Mais pour y arriver il faut bien faire croître les vitesses et en conséquence passer par les régimes laminaires. Ceux-ci peuvent se produire même en marche normale (bouchage partiel, solidification localisée, etc...).

Il semblait opportun d'attirer l'attention des concepteurs sur ces phénomènes et c'est la raison pour laquelle l'étude attribue une part substantielle à l'examen du régime laminaire que personne ne souhaite réaliser.

Il est bien évident par ailleurs que les effets néfastes qui viennent d'être signalés sont atténués par la conduction dans le tube lui-même qu'il conviendrait donc de choisir, au vu de ce seul critère, comme épais et conducteur.

A ce point de vue les chaudières classiques sont mieux placées que les applications solaires dans lesquelles le fluide non pressurisé n'exige pas de résistance donc pas d'épaisseur de la part des tubes tandis que les questions de corrosion font préférer dans le cas du solaire des aciers inoxydables, moins conducteurs que les aciers ordinaires.

Le travail effectué n'examine pas tous les cas de figures, son but étant de prévenir les utilisateurs et de calculer quelques cas parmi les plus typiques. De ce fait la prospective des recherches pour rendre la connaissance cohérente et complète sur ces types de problèmes découle presque automatiquement de ce qui vient d'être exposé.

Il s'agit de :

- l'extension des calculs dans le cas du régime turbulent en tenant compte de l'établissement du régime thermique bien que celui-ci s'effectue sur une longueur très courte.

- un flux radiatif mais en incidence par rapport au plan moyen formé par une batterie de tubes;

- des tubes non plus adjacents mais réunis par des ailettes. C'est le cas général des «murs rideau » des grosses chaudières modernes. Le positionnement de ces ailettes peut être utilisé pour obtenir une répartition de température plus homogène dans les tubes. A cette fin, la disposition usuelle des ailettes dans le plan de l'axe des tubes n'est pas la solution optimale. Un décalage vers la partie non chauffée des tubes pourrait diminuer les contraintes thermiques.

Un prochain article donnera les résultats relatifs à ces extensions.

\section{Bibliographie}

[1] REYNOLDS, W. C., « Turbulent heat transfer in a circular tube with variable circumferential heat flux ". Int. J. Heat Mass Transfer 6 (1963) 445.

[2] Gartner, D., Johannsen, K. and Ramm, H., « Turbulent heat transfer in a circular tube with circumferentially varying thermal boundary conditions" Int. J. Heat and Mass Transfer 17 (1974) 1003.

[3] Manohar, R., « Analysis of laminar flow heat transfer in the entrance region of circular tubes $"$. Int. J. Heat Mass Transfer 12 (1969) 15.
[4] TeST, F. L., « Laminar flow heat transfer and fluid flow for liquid with temperature dependent viscosity ». J. Heat Transfer (1968) 385.

[5] Stein, R. P., « Liquid metal heat transfer ». Adv. Heat Transfer (1966) 111.

[6] Ness, A. J., «Solution of equations of a thermal network on a digital computer ». Sol. Energy 3 (1959) 37.

[7] Debiane, M., Contribution à l'étude du transfert thermique par convection, conduction et rayonne- 
ment dans un tube soumis à un flux radiatif non uniforme. Thèse de $3 \mathrm{e}$ Cycle, Université de Poitiers (1982).

[8] ABILlon, Travaux en cours à Pointe-à-Pitre sur les capteurs à tube transparent et fluide semi-transparent.

[9] Stekelorom, P. et Soufiani, A., Comparaison entre la peinture Pyromark 2500 et un acier 316 oxydé. Application à la chaudière de Thémis. Rapport pour E.D.F., février 1982.
[10] SoufIANI, A., Etude radiative d'une chaudière à cavité cubique d'une centrale à tour. Rapport de stage E.C.P., Geser 13 (1980).

[11] SiNGH, « Heat transfer by laminar flow in a circular tube ». A.S.R. Section A 7 (1958).

[12] Graetz, L., Ann. Phys. Chem. 25 (1885).

[13] Kreith, F., Radiation Heat Transfer (International Text Book Company) Scranton. Pennsylvania 1962. 